Technique, économie et politique entre la France et l'Angleterre (XVIIe-XIXe siècles)

\title{
Liliane Pérez
}

\section{(2) OpenEdition \\ Journals}

Édition électronique

URL : http://journals.openedition.org/dht/1245

DOI : $10.4000 /$ dht. 1245

ISSN : 1775-4194

Éditeur :

Centre d'histoire des techniques et de l'environnement du Cnam (CDHTE-Cnam), Société des élèves du CDHTE-Cnam

Édition imprimée

Date de publication : 1 décembre 2010

Pagination : $9-29$

ISBN : 978-2-9530779-5-7

ISSN : $0417-8726$

Référence électronique

Liliane Pérez, «Technique, économie et politique entre la France et l'Angleterre (XVIle-XIXe siècles) », Documents pour l'histoire des techniques [En ligne], 19 | 2e semestre 2010, mis en ligne le 21 juin 2011 consulté le 21 septembre 2020. URL : http://journals.openedition.org/dht/1245 ; DOI : https://doi.org/ 10.4000/dht.1245 


\title{
Technique, économie et politique entre la France et l'Angleterre (XVIIe-XIXe siècles)
}

\author{
Liliane Pérez \\ Université Paris VII - Paris Diderot \\ EA337 Identités-Cultures-Territoires
}

O util majeur de la pratique historienne, le comparatisme a le plus souvent conduit à hiérarchiser les sociétés, à indexer l'avance économique et technique, participant $d$ ' « une vision unifiante, soit linéaire soit, au contraire, dialectique, d'une histoire que scandent les étapes à la fois nécessaires et successives du développement des sociétés », comme le pointait Maurice Aymard ${ }^{1}$; il a plus rarement été utilisé comme révélateur, pour interroger les catégories de référence, pour définir ce qui fait l'identité de chacune, préciser la part des « influences réciproques » et "faire apparaître quelque chose qui échappait à l'observation et à l'intelligence $»^{2}$.

C'est cette deuxième approche qui nous a guidés dans ce volume collectif, issu d'un colloque tenu en décembre 2006 à l'École des hautes études en sciences sociales, au Conservatoire national des arts et métiers et à la Cité des sciences et de l'industrie, dans le cadre du GDR France-lles britanniques. En effet, loin du comparatisme normatif qui a longtemps présidé à l'histoire économique et technique du couple franco-anglais, les études récentes mettent en valeur l'intensité des liens entre les deux pays, la complexité des circulations et l'intrication des pratiques d'échange et de traduction avec les représentations, les récits et les mythes développés par les contemporains entre les deux pays. Nous ne cherchons donc pas à reprendre l'étude comparée des indicateurs économiques, la mesure de l'écart et du

1 Maurice Aymard, "Histoire et comparaison », dans Hartmut Atsma et André Bruguière éd., Marc Bloch aujourd'hui, Paris, EHESS, 1990, pp. 271-278, p. 272.

2 Lucette Valensi, "Retour d'Orient. De quelques usages du comparatisme en histoire ", dans H. Atsma et A. Bruguière éd., Marc Bloch aujourd'hui, op. cit., pp. 307-322, p. 316, 322 : "Marc Bloch illustre cette opération par le cas où un fait relevé dans l'histoire de l'Angleterre - I'enclosure en l'occurrence permet d'inventer un fait homologue passé inaperçu en France ». rattrapage et la détermination d'une voie optimale de la croissance. Nous privilégions l'expérience francoanglaise des acteurs, la manière dont les contemporains ont construit sur le long terme cet espace relationnel trans-Manche, à la fois zone de transit pour des produits, des équipements et des migrants, et espace culturel où circulent des savoirs et où s'élaborent, à travers des jeux de miroirs, des constructions identitaires, des idéologies et la compréhension de réalités techniques et économiques nouvelles. L'évolution du comparatisme franco-anglais est à ce titre un bon exemple du tour de plus en plus pragmatique et expérimental, "technologique », que revêt l'histoire. Nous avons choisi de rapprocher pour révéler, de concevoir le comparatisme comme un raisonnement analogique, comme une "pensée par cas $»^{3}$, permettant l'exercice de l'objectivité - une naturalisation du comparatisme, une enquête empirique à partir de la nature des faits, de leurs particularités.

C'est pourquoi notre propos est double. Nous faisons, d'une part, le choix d'une histoire des techniques intégrée, globale, non réductible à la linéarité des avancées et des retards, mais soucieuse de restituer la complexité des appropriations et des hybridations ainsi que l'impact des marchés, de la division internationale du travail, des prélèvements de richesses et des accumulations de capital humain et symbolique, entre France et Angleterre et à l'échelle du monde, des colonies, des empires dont on sait de mieux en mieux le rôle crucial dans l'essor industriel. Patrick O'Brien a soutenu récemment que dans le domaine des techniques cotonnières, l'insertion de la Grande-Bretagne dans les échanges mondiaux, de Cromwell à Nelson, la puissance de sa flotte et celle de ses marchands ont été plus déterminantes

3 Jean-Claude Passeron et Jacques Revel éd., Penser par cas, Paris, EHESS, collection Enquête, 2005. 


\section{Technique, économie et politique entre la France et l'Angleterre (XVIIe-XIXe siècles)}

que la promotion de l'inventivité dans la culture des Lumières ${ }^{4}$. Ces techniques sont le résultat d'un pouvoir économique " global ». Si des pans de I'histoire francoanglaise ont pu pâtir du déclin de l'histoire économique et sociale ${ }^{5}$, l'analyse des circuits, des migrations et des recompositions entre centre et périphérie est particulièrement dynamique de nos jours ${ }^{6}$, et le doit en partie à l'élargissement des points de vue en histoire des techniques, aux interrogations sur les provenances et les identités des procédés, des produits, des savoirs et des passeurs. Nous y reviendrons. Notons seulement que les marchés sont devenus un lieu d'intersection de I'histoire des techniques et de l'histoire économique.

D'autre part, nous nous inscrivons dans une histoire culturelle de l'industrialisation, en termes d'imaginaires sociaux et nationaux et d'intellection du réel. Le couple franco-anglais joue un rôle fondamental dans l'émergence des représentations du progrès, des débats et des opinions adverses sur les transformations à l'œuvre. La vitalité des recherches actuelles sur ce thème vient utilement remanier un héritage historiographique déjà très riche. Nous pensons notamment aux publications des actes de quatre importants colloques: La formation des ingénieurs en perspective. Modèles de référence et réseaux de médiation, $X V I I I^{e}-X X^{e}$ siècles, par Irina Gouzévitch, André Grelon et Anousheh Karvar (2004), La France et I'Angleterre au XIXe siècle. Échanges, représentations, comparaisons par Sylvie Aprile et Fabrice Bensimon (2006), Les idées passent-elles la Manche? Savoirs, représentations, pratiques (FranceAngleterre, $X^{e}-X X^{e}$ siècles) par Jean-Philippe Genet et François-Joseph Ruggiu (2007), Charles Dupin (1784-

4 Patrick O'Brien, « Geopolitics of a global industry. Eurasian divergence and the mechanization of cotton textile production in England ", dans Giorgio Riello et Prasannan Parthasarathi éd., The spinning world. A global history of cotton textiles, 1200-1850, Oxford, Oxford university press (Pasold), 2009, pp. 351-365. Cette idée rejoint celle de William Hardy, montrant I'association établie entre l'industrie cotonnière, la puissance navale, la dette nationale et la monarchie protestante dans The origins of the idea of the Industrial Revolution, Oxford, Trafford Publishing, 2006, cite par Christine MacLeod, Heroes of invention. Technology, liberalism and British identity 1750-1914, Cambridge, Cambridge university press, 2007, p. 64.

5 Fabrice Bensimon, Robert Tombs, "Introduction», Revue d'histoire XIX siècle, 37, L'ère victorienne revisitée, 2008/2, pp. 7-11, p. 8.

6 Pour une vue synthétique de l'histoire économique comparative entre la Grande-Bretagne et l'Europe: Leandro Prados de la Escosura éd., Exceptionalism and industrialism. Britain and its European rivals, 1688-1815, Cambridge, Cambridge university press, 2004.
1873). Ingénieur, savant, économiste, pédagogue et parlementaire du Premier au Second Empire par Carole Christen et François Vatin (2009).

L'ambivalence entre la hantise de l'impérialisme et l'admiration pour une nouvelle société «industrieuse » que suscite la puissance économique anglaise au sortir de l'Empire est à l'origine de la notion de "révolution industrielle ${ }^{7}$, forgée sur le continent, d'inspiration libérale et radicale avant 1840, puis socialiste - dans tous les cas, le résultat de débats et de luttes politiques, plus que l'observation de réalités économiques ${ }^{8}$. On pourrait dire que dès le XVIII siècle, une lecture politique des faits économiques trans-Manche s'affirme, comme le note Renaud Morieux à propos des migrations d'ouvriers français ("Les débats économiques qui font rage, au sein des serviteurs de la monarchie [...], contaminent la lecture qui est faite des migrations ouvrières »). Au début du XIXe siècle, au Conservatoire royal des arts et métiers, Jean-Baptiste Say, et dans une moindre mesure, Charles Dupin, parce qu'ils sont mus par la contestation de l'ordre aristocratique, se font les apologues du machinisme anglais et de l'industrialisme. Alex Werner illustre clairement ce propos à partir des écrits de Dupin sur le port de Londres, admiré pour ses installations comme pour sa gestion par des compagnies privées (non sans masquer les échecs techniques, les accidents) ${ }^{9}$. Mais

7 Donald C. Coleman, Myth, history and the Industrial Revolution, Londres, Hambledon Press, 1992; François Crouzet, De la supériorité de l'Angleterre sur la France, Paris, Perrin, 1985; David S. Landes, «The fable of the dead horse, or, the Industrial Revolution revisited », dans Joël Mokyr éd., The British Industrial Revolution: an economic perspective, Boulder (Col.), Westview press, 1993, pp. 128-159.

8 François Jarrige, Au temps des tueuses de bras. Les bris de machines à l'aube de l'ère industrielle, Rennes, PUR, 2009; Gareth Stedman Jones, La fin de la pauvreté? Un débat historique (2004), Paris, Ėre, 2007, chap. 4 et 5 ; id., "The genesis of the 'Industrial Revolution': Jean-Baptiste Say and the French debate on industrie » et Julien Vincent, "Cycle ou catastrophe ? L'invention de la 'révolution industrielle' en GrandeBretagne, 1884-1914 », dans Jean-Philippe Genet et FrançoisJoseph Ruggiu éd., Les idées passent-elles la Manche? Savoirs, représentations, pratiques (France-Angleterre, $X^{e}-X X$ siècles), Paris, Presses de la Sorbonne, 2007, respectivement pp. 211233, 235-268. Voir aussi les contributions aux ouvrages suivants, Sylvie Aprile et Fabrice Bensimon éd., La France et l'Angleterre au XIXe siècle. Échanges, représentations, comparaisons, Paris, Créaphis, 2006 et Carole Christen et François Vatin éd., Charles Dupin (1784-1873). Ingénieur, savant, économiste, pédagogue et parlementaire du Premier au Second Empire, Rennes, PUR (coll. Carnot), 2009.

9 Sur ce thème, voir aussi : Benoît Agnès, « Le passeur des deux rives? La Grande-Bretagne dans l'action politique de Charles Dupin, 1814-1835 », dans C. Christen et F. Vatin éd., Charles 
Giorgio Riello et Patrick O'Brien rappellent qu' « avant le milieu du XIX siècle, I'économie britannique n'est pas considérée comme un modèle à suivre pour le reste de l'Europe », comme l'atteste l'intérêt des analystes pour les conséquences plus que pour les origines du succès anglais ; I'influence du radicalisme explique cette place ambiguë de l'Angleterre comme "modèle et contre-modèle " ${ }^{10}$, et la recherche d'un "productivisme à la française » (ainsi chez Jérôme Adolphe Blanqui, successeur de Say au Conservatoire $)^{11}$. De plus, le " paradigme luddite », selon I'expression de François Jarrige, notamment dans la presse napoléonienne, alimente le stéréotype péjoratif de l'ouvrier anglais, non sans « renforcer la critique de la voie anglaise de l'industrialisation $»^{12}$. C'est dans la deuxième moitié du siècle que le ton change ; la Grande-Bretagne apparaît à certains comme un exemple à suivre en termes de compromis social et de reconnaissance syndicale ${ }^{13}$.

De son côté, l'Angleterre victorienne célèbre le génie inventif (notamment par l'action des associations professionnelles de mécaniciens et d'ingénieurs et des syndicats ouvriers) ${ }^{14}$ et depuis le début du siècle, la «question des machines » - leur justification - est au cœur de l'économie politique naissante ${ }^{15}$. Pourtant, le concept de "révolution industrielle » ne s'impose chez les analystes outre-Manche qu'à la fin du XIXe siècle et

Dupin (1784-1873), op. cit., p. 53-65.

$10 \mathrm{~F}$. Vatin, "Modèle et contre-modèle anglais de Jean-Baptiste Say à Eugène Buret : révolution industrielle et question sociale (1815-1840) », dans S. Aprile et F. Bensimon éd., La France et I'Angleterre au XIXe siècle, op. cit., p. 69-88.

$11 \mathrm{~F}$. Démier, "Adolphe Blanqui: la leçon anglaise d'un économiste libéral français », dans $S$. Aprile et $F$. Bensimon éd., La France et l'Angleterre au XIX siècle, op. cit., pp. 57-59; id., «Adolphe Blanqui, un 'Libéral critique' à la chaire d'Économie politique du Conservatoire des arts et métiers », Cahiers d'histoire du Cnam, 2, 1993, pp. 59-86, p. 71.

12 François Jarrige, "Autour des résistances ouvrières au machinisme dans le secteur textile: paradigme luddite et anglophobie ouvrière dans la première moitié du XIXe siècle ", dans $S$. Aprile et F. Bensimon éd., La France et l'Angleterre au XIXe siècle, op. cit, pp. 335-350 ; id., Au temps des « Tueuses de Bras », op. cit., pp. 543-547.

13 F. Démier, "Adolphe Blanqui : la leçon anglaise », op. cit., p. 66 ; F. Vatin, "Modèle et contre-modèle anglais », op. cit., p. 88 ; lowerth Prothero, "Co-operation, liberalism and the labour and social questions in England and France C. 18501870 », dans S. Aprile et F. Bensimon, La France et l'Angleterre au XIX siècle, op. cit., pp. 159-176.

14 C. MacLeod, Heroes of invention, op. cit., chaps. 4, 10.

15 Maxine Berg, The machinery question and the making of political economy, 1815-1848, Cambridge, CUP, 1980 ; Vincent Bourdeau, François Jarrige, Julien Vincent, Les luddites. Bris de machines, économie politique et histoire, Maison-Alfort, Éditions Ère, 2006, pp. 67-81. comme un « 'lieu de mémoire' contesté » de l'identité nationale britannique ${ }^{16}$. Sur ce point, notons que des divergences d'interprétation existent. Christine MacLeod date des années 1850, marquées par la glorification des inventeurs, l'émergence de la notion de "révolution industrielle » chez les historiens Whig - une histoire ensuite effacée par la critique pessimiste du machinisme ${ }^{17}$. Pour G. Riello et P. O'Brien, les historiens sont maintenant conscients que les archives qu'ils utilisent du côté anglais, plus silencieuses que celles du continent, sur la conscience d'une révolution industrielle, « sont le produit des préoccupations contemporaines pour construire un système politique doté d'un cadre législatif et institutionnel afin d'assurer l'ordre, la stabilité et une dose de justice sociale à une époque de transition économique que I'on ne considère plus comme révolutionnaire ou cataclysmique ${ }^{18}$. C'est au prix de détours que l'idée de « révolution industrielle » serait adoptée en GrandeBretagne, mais cela n'exclut pas sa revendication côté anglais, comme le montre Marie-José Durand-Richard dans son article intitulé "Le regard français de Charles Babbage sur 'le déclin de la science en Angleterre' ». Elle rappelle ainsi l'insertion du mathématicien dans des réseaux Whig et anglicans libéraux qui entendent prendre appui sur la refonte des institutions de la science née de la Révolution française, pour réformer « le fonctionnement aristocratique de la Royal Society » et des universités, et «sensibiliser la classe dirigeante traditionnelle à l'importance nouvelle du monde industriel». L' « effort d'adaptation des institutions du savoir aux effets de la révolution industrielle », en Angleterre, passe par I'introduction des méthodes françaises, en termes de politique de la science ( $d$ 'inspiration baconienne et utilitariste), de rationalisation du travail savant (à l'instar de la « division du travail » instaurée par Gaspard Riche de Prony pour les tables du Cadastre, en 1791) et du calcul analytique laplacien, dont on sait le rôle crucial dans « la montée en régime d'une logique des opérations » chez

16 J. Vincent, « Cycle ou catastrophe ?», op. cit., p. 236

17 « With the Whig apology of steam, a new history of Britain had been sanctioned. The notion of the industrial revolution (if not the precise term) was firmly established during the middle decades of the nineteenth century "; C. MacLeod, Heroes of invention, op. cit., p. 143. Le chapitre 5 est intitulé «Watt, inventor of the Industrial Revolution ».

18 Voir aussi : Giorgio Riello et Patrick O’Brien, «The future is another country: offshore views of the British Industrial Revolution », Journal of historical sociology, 22/1, 2009, pp. 1-29 ; V. Bourdeau, F. Jarrige, J. Vincent, Les luddites, op. cit., pp. $69-70$; G. S. Jones, « Saint-Simon and the liberal origins of the socialist critique of Political Economy », dans S. Aprile et F. Bensimon éd., La France et I'Angleterre au XIX siècle, op. cit., p. 21-47, p. 29. 


\section{Technique, économie et politique entre la France et l'Angleterre (XVIIe-XIXe siècles)}

les ingénieurs français ${ }^{19}$. On retrouverait un tel détour par la France et ses techniciens héroïques affrontant l'Ancien Régime aristocratique, dans le journal libéral radical Mechanic's Magazine, fondé notamment par Thomas Hodgskin, qu'analyse Ben Marsden dans ce volume.

Au tournant du XIX' siècle, dans un autre domaine, la science historique, c'est encore à l'occasion d'un détour, la parution et le succès du livre de Paul Mantoux, La Révolution industrielle en Angleterre au XVIII' siècle en 1905, que les universitaires anglais accorderont définitivement une place privilégiée à la révolution industrielle dans le récit historique ${ }^{20}$. Non sans ironie, Gareth Stedman Jones remarque que la notion de "révolution industrielle » chez Toynbee est venue recouvrir la Révolution française et son impact en Angleterre dans les années 1790, ainsi " passés sous silence » dans l'explication des changements sociaux intervenus au cours du XIXe siècle ${ }^{21}$.

Retrouver les discours des contemporains sur les transformations qu'ils observent de part et d'autre de la Manche, restituer le sens qu'ils donnent à ces réalités selon leurs cultures nationales, selon leurs appartenances sociales et culturelles, identifier les influences, les emprunts ou les blocages, c'est accéder aux sources qui ont sous-tendu durablement le récit historique depuis la fin du XIXe siècle et comprendre les relectures dont il a fait l'objet.

Nous voudrions ajouter un troisième thème. À I'intersection de ces deux dimensions qui construisent l'espace franco-anglais (les échanges et les représentations), se tiennent les pratiques comparatives des contemporains, celles qui président à l'acquisition de savoirs techniques (la collecte, l'emprunt, la transposition), et celles qui sous-tendent l'évaluation des avantages, la formation des opinions sur l'une et l'autre nation ou la prise de décision. Le colloque qui a donné lieu à ce volume a donc aussi eu pour but d'étudier les comparaisons nées sous la plume des contemporains, les niveaux d'analyse que recouvrent cet exercice et les enjeux qui lui sont assignés. Quelle est la portée, aux yeux des historiens de l'économie et des techniques, de I'affirmation d'une pensée comparative, analogique et évaluatrice, qui, nourrie sur trois siècles, atteint sa maturité dans la première moitié du XIXe siècle ?

Avant de détailler les thèmes abordés dans cet ouvrage, insistons sur la comparaison franco-anglaise et

19 Antoine Picon, L'invention de l'ingénieur moderne. L'École des Ponts et Chaussées 1747-1851, Paris, Presses de l'École nationale des ponts et chaussées, 1992, pp. 302-307.

20 J. Vincent, "Cycle ou catastrophe? », op. cit., p. 261 ; JeanJacques Salomon, "Paul Mantoux et la Révolution industrielle », Cahiers d'histoire du Cnam, 2, 1993, pp. 121-154.

21 G. S. Jones, La fin de la pauvreté ?, op. cit., pp. 165-166. sur le changement de perspective. C'est la transformation des termes de la comparaison entre France et GrandeBretagne qui est à l'origine des travaux actuels présentés dans ce colloque.

\section{La révolution industrielle à l'épreuve du comparatisme franco-anglais}

Les comparaisons entre les deux pays constituent un héritage historiographique construit sur un siècle au moins, un thème né avec I'histoire industrielle. L'apologie du modèle anglais, I'histoire des transferts de techniques anglaises sur le continent et celle des rythmes de leur adoption ont formé un topos de l'histoire de la révolution industrielle. Celle-ci était intrinsèquement liée à l'affirmation de la "supériorité » anglaise, un concept souvent nourri de discours déterministes, tendus entre sacralisation de l'invention et dénonciation des méfaits du machinisme, réduisant de fait l'espace intellectuel de I'analyse comparée, comme l'avait montré Jean-Claude Perrot décrivant la mise en place ancienne de cette rhétorique, depuis la Révolution française ${ }^{22}$.

Toute relecture de la notion de révolution industrielle a en retour transformé le comparatisme franco-anglais. Les premiers coups de boutoir sont venus de l'hypothèse gradualiste. Les comptabilités nationales, la constitution de données agrégées macro-économiques sur les revenus par tête et sur la productivité du travail ont formé le socle des révisions quantitatives de la croissance anglaise. La mise à distance des récits fondateurs, devenus objets d'étude, s'est accompagnée d'un puissant mouvement de relecture de l'industrialisation, dessinant une analyse alternative, attentive au rôle de la demande et à l'essor des marchés (et non seulement aux traditionnels facteurs de l'offre), à la complexité des modes de production et à la pluralité des voies de la croissance ${ }^{23}$, incitant in fine

22 Jean-Claude Perrot, "Les effets économiques de la Révolution. Trente années de bilan (1795-1825)», dans id., Une histoire intellectuelle de l'économie politique (XVIIe-XVIII' siècle), Paris, EHESS, 1992, pp. 441-469.

23 Patrick Verley, L'échelle du monde, Essai sur l'industrialisation de l'Occident, Paris, Gallimard, 1997, chapitre III « A la recherche d'une typologie impossible »; id., "La révolution industrielle anglaise (note critique)», Annales ESC, 1991/3, pp. 735-755; Patrick O'Brien, Caglar Keyder, Economic growth in Britain and France, 1780-1914. Two paths to the twentieth century, Londres, Allen and Unwin, 1978 ; Roderick Floud, Donald N. McCloskey éd., The economic history of Britain since 1700, vol. 1 : 17001860, Cambridge, CUP, 1981 ; Edward A. Wrigley, Continuity, chance and change. The character of the industrial revolution in England, Cambridge, CUP, 1988 ; Maxine Berg, The age of manufactures 1700-1820, Londres, Fontana, 1985 ; Pat Hudson éd., Regions and industries. A perspective on the industrial revolution in Britain (1989), Cambridge, CUP, 1993. Pour une 
à ne pas confondre l'essor économique anglais avec la seule industrialisation ${ }^{24}$. Cette approche critique, née de la rupture historiographique instaurée par J. H. Clapham et Abbott Payson Usher dans I'Entre-deux-guerres ${ }^{25}$, revêtait une double portée. Le comparatisme francoanglais en a été subverti.

D'une part, l'écart des indicateurs de croissance entre la France et l'Angleterre était révisé à la baisse ${ }^{26}$. Les analyses quantitatives contribuaient à minimiser I'ampleur des transformations survenues lors de la révolution industrielle. D'autre part, cet apport se doublait d'une conclusion qualitative. Si les taux de croissance étaient proches, entre France et Angleterre, c'est que des structures de production différentes pouvaient, dans des contextes particuliers, revêtir une efficacité propre ${ }^{27}$ (une « rationalité » propre, disait-on). Au modèle de la supériorité, succédait celui des voies de la croissance, étroitement lié au succès de la notion de dépendance du sentier, de path dependency chez les économistes. Reconnaître la singularité des expériences permettait d'identifier le caractère exceptionnel anglais sans pour autant conclure à une voie optimale de la croissance ${ }^{28}$.

Ce changement de regard a été fondamental en

synthèse récente, voir : Julien Vincent, "Industrialisation et libéralisme au XIXe siècle: nouvelles approches de I'histoire économique britannique », Revue d'histoire XIXe siècle, 37, L'ère victorienne révisitée, 2008/2, pp. 87-110.

24 Gareth Stedman Jones, "Repenser le chartisme », Revue d'histoire moderne et contemporaine, 2007, vol. 54-1, pp. 7-68, pp. 9-11. Voir aussi en ce sens les débats sur l'économie londonienne: id., " L'importance de Londres dans l'histoire de la Grande-Bretagne contemporaine », Genèses, 1990, 1, pp. 47-57; Martin J. Daunton, «Industry in London : revision and reflections», London journal, 1996, 21-1, pp. 1-8; Paul Johnson, «Economic development and industrial dynamism in Victorian London », London journal, 1996, 21-1, pp. 2737; David R. Green, "The nineteenth-century metropolitan economy: a revisionist interpretation », London journal, 1996, 21-1, pp. 9-24.

25 P. Verley, L'échelle du monde, op. cit., pp. 38-41; Arthur P. Molella, "The Longue durée of Abbott Payson Usher. A P. Usher, A history of mechanical inventions », Technology and culture, 2005, vol. 46-4, pp. 779-796. Pour une synthèse, voir également : J. Vincent, "Industrialisation et libéralisme au XIX siècle », op. cit.

26 Maxine Berg, «Revisions and revolutions: technology and productivity change in manufacture in eighteenth-century England ", dans Peter Mathias, John A. Davis éd., Innovation and technology in Europe from the eighteenth-century to the present day, Oxford, Basil Blackwell, 1991, pp. 42-64 ; Patrick Verley, La révolution industrielle 1760-1870, Paris, Gallimard, 1997.

27 L. Prados de la Escosura, «Introduction: was British industrialisation exceptional? », op. cit.

28 P. O'Brien, C. Keyder, Economic growth in Britain and France, op. cit. histoire des techniques. Poser la question des rythmes de la croissance, c'était ouvrir la possibilité d'une histoire éloignée des success stories, d'une histoire marquée par la lenteur des transformations, par leur inachèvement, par la coexistence plus que par la succession de générations techniques, dans un monde du travail largement dominé par la répétition, par la maîtrise des gestes acquis dans la durée, un monde où l'innovation radicale n'offrait pas toujours d'avantages comparatifs ${ }^{29}$. Le cheminement des techniques en société, leur validation par des communautés humaines devenaient des paramètres essentiels pour les historiens, privilégiant ainsi l'étude des sites de production et des logiques propres aux économies locales, par rapport au récit linéaire, vu comme incapable de restituer la discordance des temps qui constitue la marque des savoirs techniques. Les notions d'avance et de retard perdaient leur sens, au profit d'approches ancrées dans des territoires et des communautés. Dans le domaine clé de la sidérurgie, en France, Serge Benoît, à la suite de Denis Woronoff, a montré que le maintien et la sophistication de l'énergie hydraulique, surtout dans l'affinage "à l'anglaise » (au coke), « ne fut pas le symptôme d'un archaïsme, [...], mais l'expression d'une différence foncière de structures, de marchés et de cultures techniques », favorisant " la diversité et l'extrême spécialisation des établissements $»^{30}$. Nous reviendrons sur le thème des choix techniques en évoquant la fine analyse des techniques de la vapeur que propose Alessandro Nuvolari dans cet ouvrage.

Au prisme des hypothèses gradualistes, le rôle phare de l'Angleterre était amoindri. Certains ont parlé d'une "dénationalisation » de la révolution industrielle anglaise. Les critiques ont été vives : définition restrictive des activités économiques, absence de prise en compte d'activités non comptabilisables, notamment de I'innovation en milieu artisanal, effacement des données régionales, et donc de l'intensité des transformations dans certains sites, au profit d'une vision continuiste et aplanie (pacifiée aussi) de la révolution industrielle ${ }^{31}$. Loin

29 Denis Woronoff, "Le quotidien des techniques: de la répétition aux aménagements », dans Dominique Barjot, Michèle Merger éd., Les entreprises et leurs réseaux. Hommes, capitaux, techniques et pouvoirs, XIXe-XXe siècle, Paris, Presses de l'université Paris-Sorbonne, 1998, pp. 785-791.

30 Serge Benoit, "Le rôle de l'énergie hydraulique dans la sidérurgie française au XIXe siècle : un essai de réévaluation», dans Jean-François Belhoste, Serge Benoît, Serge Chassagne, Philippe Mioche éd., Autour de l'industrie, histoire et patrimoine. Mélanges offerts à Denis Woronoff, Paris, CHEFF, 2004, pp. 24-46. 31 Pat Hudson, The industrial revolution, Sevenoaks-New York, Routledge, 1992 ; Maxine Berg, Pat Hudson, «Rehabilitating the industrial revolution », Economic history review, 45, 1992, pp. 24-50. 


\section{Technique, économie et politique entre la France et l'Angleterre (XVIIe-XIXe siècles)}

de rompre avec le modèle historiographique classique, la critique à la baisse de la révolution industrielle confortait le rôle leader du coton, du fer et du charbon et ne modifiait en rien la compréhension globale des processus de croissance, indexés sur la productivité des équipements lourds et le degré de mécanisation du travail.

Cette impasse a suscité une « réhabilitation» de la révolution industrielle, dont I'article de Maxine Berg et de Pat Hudson a donné le coup d'envoi ${ }^{32}$. Une autre image s'est dessinée: celle d'un bouleversement massif de l'organisation du travail, en rien limité à la mécanisation cotonnière, au charbon et à la vapeur, mais affectant en profondeur toutes les couches du monde du travail et revêtant de multiples visages, selon les régions, selon les secteurs, selon les marchés. Aucune fabrication n'échappe à la pression des marchés et des marchands, ni en Angleterre, ni sur le continent, mais chaque ville, chaque métier, répond différemment aux sollicitations des marchands. La territorialité des techniques est sortie renforcée par ces approches, soulignant moins le conservatisme technique de larges secteurs de production que la multiplicité des voies de l'innovation, y compris au cœur des techniques dites traditionnelles. La révolution industrielle saisie à l'échelle micro-économique et par la désagrégation des données a autorisé des analogies nouvelles, entre Lyon et Birmingham, entre Sheffield et Solingen ${ }^{33}$ - entre la Cornouaille anglaise et la France, comme le montre A. Nuvolari dans ce volume. Cette approche localisée n'a pas abouti à un émiettement ; au contraire, elle a ouvert la voie à une histoire globale, en termes d'intégration de territoires et de villes à des courants d'échanges mondiaux, en termes aussi de pluralité des facteurs de croissance, en soulignant l'interdépendance des techniques avec les marchés, les institutions, les sociabilités et les modes de légitimation des savoirs ${ }^{34}$.

32 M. Berg, P. Hudson, « Rehabilitating the industrial revolution », op. cit. 33 Maxine Berg "Commerce and creativity in eighteenthcentury Birmingham », dans id. éd., Markets and manufactures in early industrial Europe, Londres, Routledge, 1991, pp. 173204 ; P. Verley, La révolution industrielle, op. cit. ; Jochen Putsch, Manfred Krause, "The cutlery industry from production to industrial museum: Solingen and Sheffield compared ", Transactions of the Newcomen society, 1994, vol. 66-1, pp. 43-52. 34 Sur la double acception de I'histoire globale, voir Caroline Douki, Philippe Minard, "Introduction», Histoire globale, histoire connectée : un changement d'échelle historiographique, Bulletin de la Société d'histoire moderne et contemporaine, Revue d'histoire moderne et contemporaine, vol.54-4bis, supplément 2007, pp. 7-21. La compréhension « globale » que propose actuellement Robert $C$. Allen est source de débat, tant les motifs économiques (coûts salariaux, prix du charbon) sont érigés en facteurs décisifs de l'innovation : Robert C. Allen, The British industrial revolution in a global perspective, Cambridge,
À l'issue de ces révisions, quantitatives et qualitatives, les comparaisons entre France et Angleterre ont donc conforté l'idée d'une pluralité d'expériences à l'échelle européenne, y compris régionale et locale, et d'une imbrication des facteurs de croissance. En délaissant l'étude comparée des indicateurs économiques, la mesure de l'écart et du rattrapage et la détermination d'une voie optimale de la croissance, le comparatisme a renforcé I'histoire comme science pragmatique des singularités, des jeux d'échelles et de la multiplicité des facteurs. Ce décloisonnement a favorisé l'analyse de la diversité des possibles, qu'il s'agisse des voies de la croissance ou de la pluralité des mondes de production ${ }^{35}$. La part de l'artisanat dans l'innovation technique a ainsi été repensée, et les catégories interrogées, notamment la spécificité de l'industrie au XVIII siècle, de plus en plus vue comme imbriquée à d'autres formes productives $^{36}$, et moins différente qu'on ne le pensait des autres entreprises, artisanales et marchandes ${ }^{37}$. À mesure que le comparatisme franco-anglais s'est détourné des ambitions normatives de classement, la révolution industrielle est donc devenue un fait d'histoire total, indissociable de transformations plus vastes de l'économie, notamment de la puissance acquise par la sphère des échanges en Angleterre. L'importance du commerce (et de l'agriculture) dans les Private Acts du Parlement au XVIII siècle le confirme, comme le rappellent Christiane Demeulenaere-Douyère et David J. Sturdy dans ce recueil; de même, la mobilisation statistique non sur l'industrie, mais sur les échanges extérieurs ${ }^{38}$, et l'importance accordée au commerce, dans

Cambridge university press, 2009. Voir l'usage très convaincant de ce modèle par Alessandro Nuvolari, dans le présent volume. 35 Robert Salais, Michael Storper éd., Les mondes de production. Enquête sur l'identité économique de la France, Paris, EHESS, 1993. 36 M. Berg, "Commerce and creativity in eighteenth-century Birmingham », op. cit. ; Pat Hudson éd., Regions and industries, op. cit. Ce thème a été fondamental dans le renouveau de I'histoire industrielle en France: Aux origines de la Révolution industrielle. Industrie rurale et fabriques, Revue du Nord, 240, janvier-mars 1979 ; Serge Chassagne, Le coton et ses patrons. France, 1760-1840, Paris, EHESS, 1991 ; Denis Woronoff, L'industrie sidérurgique en France pendant la Révolution et I'Empire, Paris, EHESS, 1984 ; Line Teisseyre-Sallmann, L'industrie de la soie en Bas-Languedoc, XVII -XVIII siècles, Paris, École des Chartes, 1995.

37 Maxine Berg, Luxury and pleasure in eighteenth-century Britain, Oxford, Oxford university press, 2005, p. 169 ; id., "Product innovation in core consumers industries in eighteenthcentury Britain », op. cit., p. 152 ; Catherine Lanoë, La poudre et le fard. Une histoire des cosmétiques de la Renaissance aux Lumières, Seyssel, Champ Vallon, 2008.

38 Philippe Minard, "La statistique industrielle en Angleterre au XVIII siècle: position du problème », dans Dominique 
la pensée économique, comme source du progrès des sciences et des arts ${ }^{39}$. La culture négociante, les pratiques de l'échange, l'intensification des circulations sont de plus en plus perçues comme cruciales dans l'élaboration d'une culture technique innovatrice aux XVIII' et XIXe siècles $^{40}$. En lien, le renouveau de l'analyse comparatiste a permis de mettre en valeur "l'expérience franco-anglaise » des acteurs, la manière dont les contemporains ont construit sur le long terme l'espace relationnel trans-Manche, au fil d'un procès d'échange qui constitue la clé de voûte des capacités techniques déployées lors de la première industrialisation.

\section{Révisions du comparatisme : échanges techniques et histoire globale}

L'étude des échanges techniques entre la France et l'Angleterre s'inscrit dans la compréhension globale des voies de l'industrialisation, à deux échelles, le monde et I'Europe.

D'une part, on l'a vu, le rôle de l'Empire britannique et du sea power est de plus en plus affirmé. L'image de la révolution industrielle est devenue indissociable d'économies internationales déjà fortement intégrées, autorisant des prélèvements de richesses (par l'esclavage, par l'exploitation de ressources naturelles) et des cycles de renouvellement des produits, des matières, des modèles grâce au dynamisme de l'économie asiatique qui, à son tour, offre des marchés en expansion aux producteurs occidentaux. Cette histoire globale et comparative a privilégié les interactions entre l'Europe et I'Asie, considérées comme le cœur du processus de modernisation occidental ${ }^{41}$. L'histoire coloniale est

Margairaz, Philippe Minard éd., L'information économique XVleXIXe siècle, Paris, CHEFF, 2008, pp. 227-239.

39 G. S. Jones, "Saint-Simon and the liberal origins », op. cit. p. 23 ; C. MacLeod, Heroes of invention, op. cit., p. 64.

40 Michel Cotte, «From trade to industry. The independant informative networks of European firms (early nineteenth century) ", ICON, 5, 1999, pp. 167-187; id., De l'espionnage industriel à la veille technologique, Belfort/Besançon, UTBM/ Presses universitaires franc-comtoises, 2005; id. éd., Les circulations techniques. En amont de l'innovation: hommes, objets et idées en mouvement, Belfort/Besançon, UTBM/Presses universitaires franc-comtoises, 2004.

41 On ne détaillera pas ici la bibliographie très dense, à la suite des ouvrages de Kirti N. Chaudhuri, David S. Landes et Kenneth Pomeranz; le lecteur trouvera des synthèses récentes dans: M. Berg, Luxury and pleasure in eighteenth-century Britain, op. cit. (chap. 2 "Goods from the East»); id., "In pursuit of luxury: global origins of British consumer goods in the eighteenth century », Past and present, 182, 2004, pp. 85-142 ; Giorgio Riello et Prasannan Parthasarathi, «Introduction. Cotton textiles in global history ", dans id. éd., The spinning world., profondément revisitée ; au modèle de la domination et de la soumission, succède celui des hybridations, des zones de contact, des frontières et de l'« import blending » selon Giorgio Riello et Prasannan Parthasarathi, réfutant la notion restrictive $d^{\prime}$ ' import substitution ».

C'est le cas en histoire des sciences ; Kapil Raj a montré que le modèle de la circulation des savoirs botaniques entre Asie et Europe ne peut se limiter à celui de l'exploitation impérialiste mais qu'il recouvre un processus actif de création de savoirs, notamment par les intermédiaires, consolidant leur propre position sociale, politique ou commerciale, au cours de multiples transactions ${ }^{42}$. L'Angleterre, en ce sens, développe une voie originale de formation des élites locales. Dans cet ouvrage, A. Werner évoque, dans le domaine des techniques portuaires, la visite de l'ingénieur indien Ardaseer Cursetjee à Londres, en 1839, son insertion dans les sociabilités techniciennes de la métropole, au profit de l'East India Company et des installations à Bombay qu'il dirige, suscitant des élans expansionnistes dans la presse anglaise.

Cette attention à la complexité des connexions marque aussi les études récentes sur le dynamisme de I'Inde textile, y compris pendant la période coloniale. Ce courant est particulièrement bien représenté par l'équipe du Centre for global history de Warwick, autour de Maxine Berg et Giorgio Riello. L'activité des marchands indiens, leurs échanges avec d'autres systèmes commerciaux en Asie, leur longue expérience des «traductions» et des hybridations dans l'économie des produits sont mis en valeur pour expliquer la suprématie des cotonnades du subcontinent dans les échanges mondiaux ${ }^{43}$. L'Inde forge le goût européen et ses produits apparaissaient comme des gages d'authenticité, alors que se profile un monde de production multicentré (bien différent des économiesmondes braudéliennes) et un jeu incessant d'imitations et de compositions (« import blending »), non sans poser la question de la désignation des produits, et plus largement, celle des identités des choses et des êtres.

D'autre part, à une historiographie fondée sur I'héroïsation du génie technique anglais et sur le rôle leader de I'Angleterre dans l'industrialisation de I'Europe,

op. cit., pp. 1-13; J. Vincent, «Industrialisation et libéralisme au XIXe siècle », op. cit.

42 Kapil Raj, Relocating modern science: circulation and the construction of knowledge in South Asia and Europe, 16501900, Basingstoke, Palgrave Macmillan, 2007.

43 Prasannan Parthasarathi, The transition to a colonial economy. Weavers, merchants and kings in South India, 1720-1800, Cambridge, CUP, 2001 ; Ruth Barnes, Textiles in Indian Ocean societies, Londres, Routledge, 2004 ; Giorgio Riello, Tirthankar Roy, Om Prakash éd., How India clothed the world. Cotton textiles and the Indian Ocean, 1500-1850, Leyde, Brill, 2009. 


\section{Technique, économie et politique entre la France et l'Angleterre (XVIIe-XIXe siècles)}

les analyses récentes substituent le caractère composite et somme toute collectif de l'effort de modernisation industrielle. C'est l'aptitude au réseau dans de larges couches du monde du travail et l'accélération des circulations à différentes échelles qui ont fait de l'Europe un « réservoir » de compétences techniques à partir duquel des voies originales de développement se sont affirmées, différentes selon les Etats, selon les régions. Les modalités de transmission de savoirs techniques, les passeurs, les supports et les régimes d'ouverture des savoirs occupent donc une place centrale dans les recherches actuelles sur l'industrialisation.

Les contributeurs à ce recueil soulignent ainsi le rôle de « creuset » joué par l'Europe pour l'industrie anglaise depuis le $X V l^{e}$ siècle. Un fait est acquis : la révolution industrielle anglaise est une « réalisation européenne » («European acheivement »), entamée de longue date et durablement poursuivie ${ }^{44}$. L'article de Michèle Virol sur la carrière anglaise de l'ingénieur fortificateur Jean Thomas formé auprès de Vauban, confirme qu'à la fin du XVIII siècle, I'Angleterre cherche en France des compétences techniques (notamment pour assurer son emprise sur I'Irlande). Ce tropisme ne s'efface pas au siècle suivant. La contribution de Renaud Morieux, sur le réseau des mulquiniers du Cambrésis qui introduisent la fabrication des toiles de lin à la fin du XVIII e siècle, dans le Sussex, constitue un nouvel exemple de la complexité des circuits techniques à cette période, en rien limités à un flux de l'Angleterre vers le continent, ce que confirme, dans un autre domaine, l'étude de Christelle Rabier sur les savoirs chirurgicaux et le topos de la supériorité parisienne au début du XIX siècle. À partir du milieu du siècle, dans plusieurs secteurs, les techniciens français expriment un patriotisme décomplexé et gagnent I'estime des Anglais, qu'il s'agisse de la fabrication d'instruments scientifiques qu'évoque Paolo Brenni, de la construction des gares ou de la gestion des eaux urbaines - les ingénieurs affirment que Paris est devenu un exemple ( $K$. Chatzis). Le rôle joué par Augustin Betancourt, véritable médiateur de la culture technologique française en Espagne et en Russie - où

44 Christine MacLeod, «The European origins of British technological predominance », dans L. Prados de la Escosura éd., Exceptionalism and Industrialism, op. cit., pp. 111-126. Christine MacLeod évoque un contexte de production « pan-européen », expression que I'on trouve également chez Roger Smith décrivant I'horlogerie londonienne, impossible à comprendre hors de réseaux européens, notamment en raison des circuits de sous-traitance organisés à l'échelle internationale: Roger Smith, "The Swiss connection. International networks in some eighteenth-century luxury trades », Journal of design history, 172, 2004, pp. 123-139. il réalise une synthèse des savoirs d'ingénierie française et britannique - I'atteste également, comme l'explique Irina Gouzévitch.

De plus, à l'opposé du modèle des «transferts » techniques, I'analyse actuelle des circulations met I'accent sur les processus de transposition, de «traduction» et de construction permanents des savoirs, sur le rôle des «objets-frontières » ${ }^{45}$. Delphine Gardey explique à propos de l'histoire de la sténographie, technique anglaise « resignifiée en France, et plus largement en Europe continentale », qu' « il est possible de parler pour la France d'une 'réinvention' de la sténographie sous la Révolution. Par réinvention nous entendons pour I'essentiel, et en dépit de l'adaptation de l'anglais, que de nouvelles utilités (et ce faisant de nouvelles significations formelles) sont attribuées aux méthodes d'écritures abrégées au cours de cette période. [...]. La traduction a lieu parce que la 'prise' est possible ». Notons que lorsque les Anglais s'intéressent aux enseignements de l'École centrale, ils demandent communication de « ses leçons sténographiées $»^{46}$.

Une telle approche, éloignée d'une « conception universaliste des sciences et des techniques », fait la part aux « négociations conflictuelles ${ }^{47}$, ainsi qu'aux freins et aux échecs, y compris en Angleterre. Alain Cottereau I'a montré en décrivant l'échec de l'introduction du métier Jacquard à Spitalfields ${ }^{48}$. La structure municipaliste et l'écheveau de négociations entre artisans et marchands lyonnais, dont le métier porte l'inscription, manquent à Londres où la gestion individualisée de l'invention via les patents ne permet pas l'investissement d'intérêt et de compétences nécessaire à son usage efficace. La territorialité de la technique l'emporte sur tout effort de traduction. L'étude d'Alessandro Nuvolari sur les débats, en Angleterre, à propos des machines à vapeur à haute-pression développées par les ingénieurs de

45 Pascale Trompette et Dominique Vinck, « Retour sur la notion d'objet-frontière », Revue d'anthropologie des connaissances 2009/1, vol. 3, n 1, pp. 5-27.

46 André Grelon, « Du bon usage du modèle étranger : la mise en place de l'École centrale des arts et manufactures », dans Irina Gouzévitch, André Grelon et Anousheh Karvar éd., La formation des ingénieurs en perspective. Modèles de référence et réseaux de médiation, XVIII'-XX' siècles, Rennes, PUR (coll. Carnot), 2004, pp. 17-21.

47 Irina Gouzévitch, André Grelon et Anousheh Karvar, "Présentation », dans id. éd., La formation des ingénieurs en perspective, op. cit., pp. 11-14.

48 Alain Cottereau, "The fate of collective manufactures in the industrial world. The silk industries of Lyon and London, 1800-1850 ", dans Charles F. Sabel, Jonathan Zeitlin éd., World of possibilities. Flexibility and mass production in Western industrialization, Cambridge, CUP, 1997, pp. 75-152. 
Cornouaille anglaise alors que ceux du Lancashire restent fidèles aux machines de Watt confirme l'inscription locale des innovations techniques et le rôle des acquis, du cheminement en société dans la construction de I'efficacité technique. La France, moins prompte à faire le choix de la vapeur, explique-t-il, ne pâtit pas d'une situation de lock-in et peut rapidement s'équiper en machines de la nouvelle génération, du fait aussi de I'intérêt scientifique soutenu pour la thermodynamique. L'échange technique a donc lieu entre la Cornouaille anglaise et la France et il est à la source d'une divergence durable dans les techniques de la vapeur entre France et Grande-Bretagne. C'est le modèle « rejeté » par les Anglais (sauf en Cornouaille) qui est adopté et développé en France. A. Nuvolari, se référant explicitement aux travaux de Paul A. David et de Robert C. Allen, offre ainsi une contribution originale à la révision du modèle de la supériorité technique britannique. Dans les années 1820, « il n'y a plus de situation de leader contre suiveur, mais simplement un choix différent de technique dicté par des environnements économiques différents ».

Ce caractère circonstancié des techniques est clairement exprimé par les experts mobilisés dans les entreprises de transfert. Konstaninos Chatzis détaille finement, dans sa contribution, les aménagements des techniques anglaises par les ingénieurs parisiens du service des eaux et note leur conscience de la singularité nationale des dispositifs. Pour l'ingénieur Jules Dupuit, «les procédés de filtration artificielle, tels qu'ils sont appliqués en Angleterre, ne peuvent être considérés que comme des solutions locales, dont il est difficile de déduire des principes généraux qui permettent de les étendre à d'autres localités avec certitude de succès... ». D'autres raisons président encore à la fermeture de communautés aux apports de l'étranger; ainsi la forte autonomie et la réputation internationale de la chirurgie française permettent « de comprendre sa faible perméabilité aux savoirs et savoir-faire étrangers », explique Christelle Rabier.

Lorsqu'elles ont lieu, les circulations de techniques, de matériaux, de produits sont indissociables du procès continu de recomposition des origines et des identités (des produits, des matières, des techniques et des hommes) ${ }^{49}$,

49 Liliane Hilaire-Pérez, Catherine Verna, « Dissemination of technical knowledge in the middle ages and the early modern history. New approaches and methodological issues », Technology and culture, vol. 47, juillet 2006, pp. 536-565, nouvelle édition " La circulation des savoirs techniques du Moyen Âge à l'époque moderne. Nouvelles approches et enjeux méthodologiques", Tracés, 16, juin 2009, pp. 25-61; Kapil Raj, «Introduction: circulation and locality in early modern science », British journal for the history of science, à paraître. Nous remercions l'auteur de elles participent de la complexification croissante des taxinomies dans une économie marquée par I'intensification des échanges et l'extension des circuits ${ }^{50}$. Reprenant une thématique qui nous avait intéressée dans le cadre des circulations de modèles juridiques dans la gestion de l'innovation, nous avons pu souligner, dans le domaine des savoirs techniques, les mouvements incessants d'appropriation entre l'Angleterre et la France au XVIII e siècle (dans les deux sens), qu'il s'agisse des liaisons entre l'industriel Matthew Boulton et les fabricants français pour acquérir les techniques de l'ormoulu (via le marchand franco-anglais et polyglotte Solomon Hyman), des transactions multiples d'Ami Argand et de Robert Howard pour la fabrication des lampes à courant d'air, ou de la production d'outillage en acier fondu de Sheffield par les Huntsman pour les Blakey de Paris, reposant une conformation des matériaux et des modèles anglais aux besoins des ouvriers parisiens ${ }^{51}$. Les outils de Sheffield (marqués «London ») sont façonnés par l'économie parisienne et par une voie d'échange construite sur deux générations entre France et Angleterre, que porte à bout de bras Madame Blakey, née Elisabeth Aumerle, mercière parisienne secondée par l'abbé Corn, jacobite du couvent des Augustines («Les dames anglaises»), pour la traduction des commandes anglaises.

Les hybridations, loin de marquer les techniques seulement, sont aussi au cœur des remodelages identitaires des intermédiaires. La mixité des appartenances est constitutive de l'identité des passeurs, ainsi des " transmigrants», « immigrants dont la vie quotidienne dépend de leurs multiples et constantes interconnections par-delà les frontières et dont les identités publiques se configurent en relation avec plus d'un État-nation » (R. Morieux). C'est le cas de l'ingénieur Jean Thomas passé en Angleterre, étudié par Michèle Virol : huguenot

\section{la communication de son travail.}

50 Jean-Yves Grenier, L'économie d'Ancien Régime. Un monde de l'échange et de l'incertitude, Paris, Albin Michel, 1996.

51 Liliane Hilaire-Pérez, «Des entreprises de quincaillerie aux institutions de la technologie : I'itinéraire de Charles-Emmanuel Gaullard-Desaudray (1740-1832) », dans J.-F. Belhoste, S. Benoît, S. Chassagne, P. Mioche éd., Autour de I'industrie, op. cit., pp. 547-565; id., « Les échanges techniques dans la métallurgie

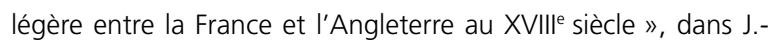
P. Genet, F.-J. Ruggiu éd., Les idées passent-elles la Manche?, op. cit., pp. 161-183; id., «Steel and toy trade between England and France: the Huntsmans' correspondence with the Blakeys (Sheffield-Paris, 1765-1769) », Historical metallurgy, 42-2, 2008, pp. 127-147 ; Liliane Pérez et Bernard Vaisbrot, "Matthew Boulton's Jewish partners between France and England. Innovative networks and merchant Enlightenment », à paraitre dans Matthew Boulton - Enterprising industrialist of the enlightement, Farnham, Ashgare, à paraître. 


\section{Technique, économie et politique entre la France et l'Angleterre (XVIIe-XIXe siècles)}

d'ascendance française, il fonde une famille en Angleterre et adopte la nationalité britannique en 1706 (sans pour autant s'intégrer facilement, sans renier son passé non plus) ; il acquiert peu à peu l'anglais et, pour conserver une position sociale, «traduit son savoir-faire militaire dans les relevés agricoles ». Un siècle plus tard, I'itinéraire de l'ingénieur Augustin Betancourt entre les Canaries, I'Espagne, la France, l'Angleterre et la Russie révèle combien son identité d'expert technique se construit au fil de ses voyages, notamment de part et d'autre de la Manche puisqu'il séjourne entre ces deux rives plus de la moitié de son temps pendant la première période de sa vie, de 1778 à 1808. I. Gouzévitch qui a analysé finement tous ses déplacements montre que la synthèse des " deux cultures techniques qui sont alors référentielles», celle française et celle anglaise, en somme «sa double culture », " fait de lui un technicien et un savant de stature assez particulière » et sous-tend sa fonction novatrice d'expert technique ainsi que sa réflexion théorique (L'essai sur la composition des machines, 1808). C'est à l'échelle européenne, et finalement en Russie - pays dans lequel le français et les traductions jouent un rôle clé dans l'établissement des savoirs technologiques ${ }^{52}$ que se lisent la carrière et la postérité de Betancourt. Indissociablement liée aux enjeux professionnels, la part de l'engagement personnel dans cette mobilité constitue en soi un mode d'existence et l'œuvre d'une vie. Les séjours « acquièrent pour lui, avec le temps, une valeur autonome », celle de la liberté et de la transgression que procure le déplacement. Ils « foisonnent d'activités, cognitives, créatives et inventives », dont I. Gouzévitch dresse la typologie. Loin de se résumer à des missions officielles, les voyages de Betancourt sont aussi des "fugues que l'homme s'évertue à déguiser en charges commissionnées », sources de rencontres multiples, d'inventions et d'entreprises (comme la télégraphie optique). Ils lui fournissent sans cesse des occasions et les moyens d'élaborer des projets, en somme la possibilité de se construire, de constituer son savoir et de penser la technique par l'action subjective ${ }^{53}$.

52 Irina Gouzévitch, De la Moscovie à l'empire russe: le transfert des savoirs européens, n spécial de Sabix, 33, 2003; id., " Le transfert technique et ses avatars. Le cas de la Russie », Documents pour l'histoire des techniques, 14, 2007, pp. 14-23; Dmitri Gouzévitch, Irina Gouzévitch, « Le Journal des voies de communication. Histoire d'une revue bilingue russe-française (1826-1842) », dans Patrice Bret, Konstantinos Chatzis, Liliane Pérez éd., Des techniques dans la presse à la presse technique, Paris, L'Harmattan, 2008, pp. 75-93.

53 Ana Cardoso de Matos, M. Paula Diogo, Irina Gouzevitch, André Grelon éd., Les enjeux identitaires des ingénieurs : entre la formation et l'action/The quest for a professional identity:
Réseaux, circuits, identités mêlées, multilinguisme, traductions et adaptation de soi : dans la «zone de contact » franco-anglaise et ses espaces de résonance, s'affirment des capacités analogiques, combinatoires, substitutives et comparatives, portées par des logiques de décloisonnement tant géographiques que sociales et identitaires - autant des creusets pour l'invention et la pensée de synthèse, tant ces situations de dialogues entre mondes hétérogènes induisent de polyvalence, de modularité et d'abstraction ${ }^{54}$, et président ainsi à l'émergence d'un « espace de la technique $»^{55}$.

Dans ce processus de construction technologique, les mobilités ont donc joué un rôle clé. L'un des enjeux du colloque était de restituer la matérialité des échanges, "les déplacements réels des hommes, des textes et des objets » (C. Rabier). De la Renaissance et jusqu'au $X I X$ e siècle, I'Angleterre reste " une terre d'immigration ouvrière » (R. Morieux) et I'on perçoit de mieux en mieux les ressorts politiques de ce marché du travail transnational en termes de définition des frontières ${ }^{56}$ et d'intégration différenciée des migrants, notamment par les guildes londoniennes ${ }^{57}$. R. Morieux, dans son article sur l'émigration des mulquiniers dans le Sussex, rappelle qu'entre répression des débauchages, difficultés de contrôle et divergences au sein des administrations, s'installe un courant migratoire "circulaire » vers I'Angleterre, composé d'ouvriers poussés par la crise textile et de "migrants structurels», qui organisent le circuit et répètent les opérations de transfert. En France, I'installation et l'accueil de la main d'œuvre anglaise est aussi analysé de façon précise actuellement, et permet par exemple une "typologie des attitudes professionnelles » face aux techniciens (et aux techniques) de l'étranger ${ }^{58}$.

Les travaux du colloque ont donc révélé une connaissance de plus en plus fine des migrants. Ce sont d'une part les colonies d'ouvriers, les techniciens expatriés. Le rôle de la religion, longtemps érigé en facteur majeur de départ et associé à des compétences particulières, est actuellement relativisé, comme l'illustrent les articles de

engineers between training and action, Lisbonne, Colibri/ CIDEHUS/CIUHCT, 2009.

54 P. Trompette et D. Vinck, « Retour sur la notion d'objetfrontière », op. cit., p. 9.

55 Hélène Vérin, La gloire des ingénieurs. L'intelligence technique du XVI ${ }^{\text {e }}$ au XVIII e siècle, Paris, Albin Michel, p. 397.

56 Renaud Morieux, Une mer pour deux royaumes : la Manche,

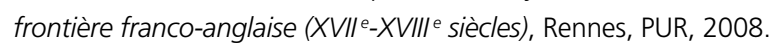
57 Lien B. Luu, Immigrants and the industries of London, 1500-1700, Aldershot, Ashgate, 2005 ; id., "Immigrants and the diffusion of skills in early modern London: the case of silk weaving ", Documents pour l'histoire des techniques, 15, juin 2008, pp. 32-42.

58 François Jarrige, Au temps des «Tueuses de Bras », op. cit. 
M. Virol et R. Morieux ${ }^{59}$. Les profils sont mieux cernés, qu'il s'agisse des travailleurs anglais en France ${ }^{60}$, ou des Français passés en Angleterre (R. Morieux peut lister les ressources foncières des mulquiniers émigrés) ; de même, leurs difficultés d'intégration (comme pour Jean Thomas, étudié par M. Virol) et la xénophobie, y compris en milieu ouvrier ${ }^{61}$. D'autre part, l'intérêt lors du colloque s'est aussi porté vers les visiteurs middle-class et les élites cosmopolites, lettrées, savantes, industrielles, formant des communautés culturelles, les unes structurées par des institutions (académies, sociétés des arts, Institution of civil engineers), les autres par des réseaux et des clientèles, ou bien unies par un habitus culturel, le voyage comme " découverte de soi et de l'autre " ${ }^{62}$. Pour certains, comme Blanqui, l'Angleterre reste une destination romantique ; le récit de voyage se nourrit d'un éclectisme, qui exprime aussi l'enthousiasme pour une société nouvelle ${ }^{63}$.

Une constante s'affirme, "I'utilité des voyages », à mesure que se déploient l'activité de renseignement et les « mobilités pédagogiques », selon l'expression de Daniel Roche. L'époque moderne connaît bien «la primauté accordée à l'observation directe sur l'interrogation à distance (la correspondance) » etce « moded'investigation auquel l'administration a recours : la mission $»^{64}$. Sylviane Llinares, étudiant les mémoires des marins français en Angleterre, souligne le rôle des voyages dans «la production d'information économique et technique dans I'Europe des Lumières ». Dans ce domaine, si la part des réseaux marchands et de leurs institutions (y compris la presse) d'un côté, si celle des personnels d'ambassades et consulaires de l'autre, ont fait l'objet d'importants travaux ${ }^{65}$, la diversité des systèmes d'information mis en œuvre par les États et leur signification rapportée à la nature des terrains d'enquête forment un sujet de

59 Sur la mise en cause de l'assignation de compétences techniques particulières à des minorités, voir le dossier " Minorités et circulations techniques du Moyen Âge à l'époque moderne (Orient, Occident) », Liliane Pérez éd., Documents pour I'histoire des techniques, 15, juin 2008.

60 Pierre-Jacques Derainne, «Les perceptions sociales des travailleurs migrants britanniques en France dans la première moitié du XIXe siècle », dans S. Aprile et F. Bensimon éd., La France et l'Angleterre au XIXe siècle, op. cit, pp. 351-367.

$61 \mathrm{lbid} .$, pp. 362-365 ; F. Jarrige, « Autour des résistances ouvrières au machinisme », op. cit., pp. 347-349.

62 Daniel Roche, Humeurs vagabondes. De la circulation des hommes et de l'utilité des voyages, Paris, Fayard, 2003.

63 F. Démier, « Adolphe Blanqui : la leçon anglaise », op. cit., pp. 51-52. 64 Antoine Savoye, "L'État enquêteur, de Guizot à Le Play ", dans id., Les débuts de la sociologie empirique, Paris, Méridiens Klincksiek, 1994, pp. 13-51.

65 D. Margairaz, P. Minard éd., L'information économique XVle$X I X$ siècle, op. cit. recherche largement ouvert. Dans le domaine maritime, S. Llinares met en valeur un milieu, celui des officiers. Ils représentent une génération et un temps particuliers de I'information navale, inscrits entre « la tradition du voyage technique » colbertiste (continuée sous Maurepas) qui a placé au premier plan les constructeurs, tels Julien Geslain et Blaise Ollivier (que Margaret Bradley évoque dans sa contribution) et la timide présence des ingénieurs de la Marine à la fin du siècle, ainsi Alexandre Forfait, célèbre pour son voyage en Angleterre en 1788 - une exception, car les ingénieurs de la Marine quittent peu les ports français, précise l'auteure. Les mémoires sur la Navy sont dus aux officiers navigants, tels les enseignes de vaisseau Henri d'Oraison et François-Étienne de Rosily-Mesros et le lieutenant de vaisseau Armand de Kersaint. Le rôle des officiers, plus que des ingénieurs, dans le réseau d'information technique, doit être rapproché de l'état de la science constructive navale, bien différente de celle des fortifications, tant s'y exprime la difficulté $d^{\prime}$ « introduire dans une même rationalité mécanique, la navigation et la construction des vaisseaux ${ }^{66}$. "Dans la construction navale, on ne dispose pas [...] de la justification théorique des choix constructifs». Comme l'a montré Hélène Vérin, dans la Marine, "I'administration des pratiques" précède "leur maîtrise technique », ce qui favorise le recours aux délibérations des charpentiers et des officiers et la «normalisation des informations pertinentes »ainsi dans les devis de campagne ${ }^{67}$. À la différence de la Guerre, ce n'est qu'à la fin du XVIII siècle qu'il revient à des « ingénieurs embarqués » le devoir de contribuer à des devis (quand ces ingénieurs acquièrent finalement le statut d'officier).

En matière de fortifications, l'insertion des ingénieurs dans les réseaux d'information est déjà largement acquise. La création de corps dans différents pays d'Europe à partir du XVIII siècle, leur offrant une stabilité professionnelle, modifie sensiblement leurs raisons de migrer, note M. Virol. Moins tenus de se déplacer pour offrir leurs services aux princes, les ingénieurs jouent dès lors un rôle central dans l'essor des déplacements pédagogiques. De même, dans le génie civil, les visites des ingénieurs s'intègrent à des logiques d'apprentissage et d'enquête, bien identifiées par Antoine Picon ${ }^{68}$. L'exemple de Betancourt, instaurant un circuit de formation pour pensionnaires espagnols à l'École des ponts et chaussées ( "l'équipe hydraulique ») (I. Gouzévitch) et celui de Jules Dupuit et des ingénieurs du service des eaux de la Ville

66 Jean-Jacques Brioist, Hélène Vérin, « Pour une histoire de la méthode de Renau d'Élissagaray », Documents pour l'histoire des techniques, 16, 2008, pp. 112-116.

67 H. Vérin, La gloire des ingénieurs, op. cit., pp. 340-343.

68 A. Picon, L'invention de l'ingénieur moderne, op. cit., pp. 114-115. 


\section{Technique, économie et politique entre la France et l'Angleterre (XVIIe-XIXe siècles)}

de Paris voyant dans ces séjours les moyens « d'une instruction positive et spéciale ${ }^{69}$ (A. Werner, K. Chatzis) confirment le propos. S'y ajoutent d'autres circulations, ainsi les déplacements d'ingénieurs en vue de former des élèves à l'étranger, comme les polytechniciens sollicités par Betancourt pour l'Institut du Corps des ingénieurs des voies de communication à Saint-Pétersbourg.

Les voyages de formation, qui participent d'un mode d'éducation éprouvé dans bien des professions, ont lieu aussi dans les deux sens. Christelle Rabier montre que le séjour d'apprentissage en France est caractéristique des échanges de savoirs chirurgicaux entre Paris et Londres. « Le voyage à Paris fait partie du bagage du praticien ambitieux qui souhaite faire carrière », tant la qualité de l'offre parisienne et la méritocratie du recrutement construisent «la distinction chirurgicale française ». Dans un autre domaine, celui des mathématiques, M.J. Durand-Richard rappelle que le mathématicien anglais George Peacock, proche de Babbage et admirateur comme lui des institutions de la science française, « accompagne plusieurs étudiants de Cambridge [en France] pour leur tour d'Europe », signant la densité des contacts « entre ces réformateurs et les membres de l'école laplacienne ». Aux marchés du travail et des connaissances, clefs de voûte des circulations techniques, s'ajoutent les sociabilités publiques et privées qui bien souvent règlent aussi les régimes d'ouverture des savoirs, le secret négocié, les stratégies de l'information (que recouvre mais n'épuise pas le terme d'espionnage). La palette des fonctions utilitaires des voyages apparaît dans toutes ses nuances chez Betancourt. I. Gouzévitch recense huit types de missions: " études (visites des sites, stages éducatifs, séjours dans les institutions, les laboratoires, les ateliers, etc.) ; inventions (descriptions, projets, modèles statiques ou opérationnels et/ou objets «grandeur nature »); travaux scientifiques (mémoires académiques et traités, avec tableaux et planches à l'appui) ; initiatives industrielles (mémoires et propositions argumentant l'intérêt de l'introduction en Espagne de diverses industries et d'essais d'exploitation) ; initiatives éducatives et organisation du travail collectif (projets d'enseignement et encadrement des stagiaires) ; commissions (acquisition d'équipements, de matériel didactique, d'instruments scientifiques, des modèles, des livres, etc.); médiation commerciale (ainsi l'agent commercial d'Abraham-Louis Bréguet); reconnaissance (collecte des informations sur les inventions et innovations d'intérêt technique, industriel et commercial, dans l'intérêt des États, des administrations et des particuliers) ». I. Gouzévitch souligne aussi la complexité

69 F. Démier, « Adolphe Blanqui : la leçon anglaise », op. cit., p. 52. des réseaux constitués par Betancourt, dosant la part des Français (mécaniciens, savants, ingénieurs d'État) et des Anglais (entrepreneurs), selon les missions et les pays auxquels il destine son action. Ainsi se dessinent d'autres pistes à explorer, les voyages d'entrepreneurs de tous horizons effectuant leur " grand tour d'Europe » (et non seulement les Français et les Anglais), dont les périples restent souvent méconnus, tout autant que les pratiques concrètes de collecte d'information - via de multiples supports, tels les échantillons - et les conditions de rédaction des relations de voyage, investies d'enjeux multiples ${ }^{70}$.

En effet, les communautés de migrants sont aussi scripturaires. M. Virol note que la conversation du vice-roi d'Irlande avec l'ingénieur Jean Thomas venu de France, en 1736, porte sur les traités d'architecture militaire. Aux « circulations des hommes » s'ajoute l'intensité des circulations d'écrits, manuscrits et imprimés. Dans la Marine, le "mémoire », caractéristique d'une technique administrative, est la forme obligée du rapport des officiers, et d'autres pratiques de l'écrit accoutument les navigants à réduire et synthétiser leur expérience, comme la tenue du journal de bord (S. Llinares). Dans le génie civil, la « collection Lesage » des mémoires sur I'Angleterre conservée à l'École des ponts et chaussées, décrite par M. Bradley, atteste la diffusion de ces pratiques rédactionnelles chez les ingénieurs. La constitution de bibliothèques dans les écoles d'ingénieurs s'accompagne de collections de modèles, notamment à partir des dessins. I. Gouzévitch rappelle que le Cabinet de machines de Madrid est fondé par Betancourt grâce à la médiation de l'équipe hydraulique, à l'École des ponts et chaussées, grâce aussi aux plans et aux croquis de Bartolomeo Surda qui assiste l'ingénieur lors de ses voyages anglais.

C. Rabier, analysant les circulations de savoirs chirurgicaux entre France et Angleterre vers 1800, montre l'importance acquise par les "échanges imprimés ». La tendance s'amplifie, en lien avec les possibilités nouvelles d'impression, et dans cette intensification se modifient aussi les contenus. Dans le domaine chirurgical, les langues vernaculaires l'emportent définitivement sur le latin. L'Angleterre se distingue, ses bibliothèques s'ouvrent tôt aux livres français et anglais, alors que ceux en latin dominent les corpus dans les inventaires

70 Corine Maitte remarque justement qu'«il est utile d'y puiser des enseignements sur les manufactures elles-mêmes, mais il faut aussi s'interroger sur les modalités de l'écriture, les raisons et les logiques des témoignages », dans «Au cœur des manufactures lainières européennes du XVIII siècle. Le voyage de Gian Batta Moccafy, 1766-1767 », Documents pour l'histoire des techniques, 18, 2009, p. 151-166. 
parisiens (non sans donner l'illusion d'une supériorité et d'une attractivité de la chirurgie française, alors que se manifeste surtout « le désir d'informations étrangères »). La Grande-Bretagne édite de plus, précocement, des périodiques chirurgicaux, dans lesquels sont annoncées les nouvelles publications, notamment étrangères, à partir des années 1790. À cet essor des productions vernaculaires, répond la multiplication des traductions de part et d'autre de la Manche, qu'analyse aussi l'auteure. Comme en écho, Ben Marsden, dans son article sur les représentations des techniques françaises outre-Manche, analyse I'ouverture de la bibliothèque de l'Institution of civil engineers aux traités et journaux technologiques français et aux traductions, de plus en plus nombreuses. "L'art de traduire», constitutif de l'espace savant des Lumières, de la recherche d'une «langue internationale de la science » à un moment d'éclatement et de dispersion des savoirs en Europe ${ }^{71}$, rencontre de nouveaux enjeux, ceux de l'élargissement des publics, en même temps que ceux de la langue technique. Marie Thébaud-Sorger rappelle que dans le domaine de l'aérostation, à la croisée de l'essor des économies culturelles et des concurrences patriotiques, les traductions sont accélérées. «Le traité de Tiberius Cavallo qui paraît en 1785 est traduit I'année suivante en français, en italien, en allemand ».

Un double mouvement se dessine. A mesure que s'intensifient les échanges, le public s'élargit, l'information devient publique, elle déborde le milieu des importateurs, des visiteurs et des agents de l'Etat (auxquels étaient réservés, par exemple, les résultats de l'Enquête du Régent, étudiée par C. Demeulenaere-Douyère et D. J. Sturdy). La question de I'espace public de l'économie et des techniques, notamment en milieu artisanal et ouvrier, est donc au cœur de ce livre. La presse, devenue un support majeur de la culture technique et industrielle ${ }^{72}$, joue un rôle clé. F. Jarrige souligne, en France, le rôle de la presse spécialisée, notamment celle qui s'adresse à un public ouvrier, dans la vulgarisation de l'économie politique anglaise, amplifiant les «projets d'une pédagogie industrielle » suscités par «le spectre des violences ouvrières ». L'auteur montre que les écrits et les modèles d'analyse circulent, que les arguments sont comparés et adaptés de part et d'autre de la Manche. A. Werner et $B$. Marsden rappellent le succès des traductions des écrits de Dupin sur l'Angleterre, leur publication régulière dans les revues mécaniciennes anglaises (dans lesquelles Dupin est loué pour savoir révéler aux Anglais leur grandeur). La

71 Roger Chartier, Pietro Corsi éd., Sciences et langues en Europe, Paris, CAK/EHESS/CNRS/MNHN, 1996.

72 P. Bret, K. Chatzis, L. Pérez éd., Des techniques dans la presse à la presse technique, op. cit. littérature technique de l'élite et les récits des observateurs français, explique B. Marsden, passent rapidement la Manche au début du XIX siècle, grâce aux traductions, via des versions abrégées ${ }^{73}$, via la presse. Les professeurs de génie civil britanniques, milieu particulièrement réceptif aux sciences appliquées françaises, encouragent l'apprentissage des langues modernes pour assurer des connaissances technologiques plus poussées (françaises) chez les ingénieurs. L'auteur montre que le mouvement n'est pas restreint aux élites. Le journal radical Mechanic's Magazine publie de nombreuses traductions d'ingénieurs français et ses lecteurs manient aussi des ouvrages en français. Les traductions de termes techniques sont commentées, critiquées. Dans cette revue où se forge une identité artisanale et mécanicienne, la traduction, assortie du multilinguisme de certains lecteurs, participe d'un effort de codification de la langue technique, hors des cercles savants, non sans débats et polémiques. L'identité technicienne radicale se noue dans ce travail plurilinguistique.

Cette multiplication des échanges économiques et techniques, les transactions aussi qui accompagnent la division du travail à l'échelle européenne, nourrissent des processus d'hybridation permanents. Plusieurs auteurs mettent en valeur la complexité des circuits entre les deux pays, et prennent leurs distances avec un modèle diffusionniste, construit au mépris des conditions locales de transmission et d'appropriation des savoirs ${ }^{74}$. Ces métissages de part et d'autre de la Manche imposent aux historiens d'interroger les identités, les localités, les appartenances, en somme de poser la question des " catégories de référence ». Dans certains cas, comme celui de la sténographie étudié par D. Gardey, I'enquête autorise un processus d'attribution ; I'auteure montre les liens entre l'écriture abrégée, la réforme de la langue et l'identité puritaine, sur le long terme. Dans l'effort de décomposition et de recombinaison que supposent cette écriture et sa lecture, filtrent des capacités à abstraire et à réduire ${ }^{75}$, nées de la pratique - une intelligence

73 Patrice Bret, «Les promenades littéraires de Madame Picardet. La traduction comme pratique sociale de la science au XVIIIle siècle », dans Pascal Duris éd., Traduire la science. Hier et aujourd'hui, Pessac, Publications de la Maison des sciences de I'homme d'Aquitaine, 2008, pp. 125-153.

74 L. Hilaire-Pérez, C. Verna, «Dissemination of technical knowledge », op. cit. ; K. Raj, "Introduction : circulation and locality in early modern science », op. cit.

75 Sur la rédaction et la réduction, voir également Hélène Vérin, «Rédiger et réduire en art: un projet de rationalisation des pratiques », dans Pascal Dubourg-Glatigny, Hélène Vérin éd., Réduire en art. La technologie de la Renaissance aux Lumières Paris, MSH, 2008, pp. 17-58. 
opératoire de la langue, associée à la miniaturisation des ouvrages, dessinant une passerelle nouvelle entre culture curieuse et savoirs technologiques. Dans l'article de B. Marsden, ce sont les traditionnels contrastes entre la France et l'Angleterre, entre une Angleterre pratique et une France théorique, qui sont soumis à relecture critique. Plusieurs contributeurs soulignent les proximités entre les cultures techniques des ingénieurs anglais et français au début du XIXe siècle. Les jeux de miroir entre les ingénieurs civils anglais et l'École centrale des arts et manufactures, inspirée du modèle anglais mais admirée à son tour outre-Manche, I'attestent ${ }^{76}$. La " recherche des rapports de complémentarités et d'exclusion » entre France et Angleterre a définitivement pris le pas sur le classement unilinéaire et sur la «typologie qui se borne à classer » les ressemblances et les différences, au mépris de « la diversité du réel $»^{77}$.

\section{Représentations franco-anglaises et imaginaire industriel}

Un deuxième ensemble de questionnements concerne l'imbrication des pratiques d'échange avec les représentations d'une part, avec les modes d'intellection des réalités d'autre part. Quelles constructions identitaires, quels classements, quelles hiérarchisations, quelles cultures comparatives, accompagnent l'intensification des circulations techniques des deux côtés de la Manche ?

Si le schéma unilinéaire d'évolution des économies est abandonné par les historiens, reste à comprendre et à périodiser l'émergence de cet argumentaire. Du début du XVIII siècle au milieu du XIXe siècle, des récits de voyage, des correspondances, des rapports d'ingénieurs, des enquêtes administratives, des articles de presse construisent l'espace culturel de la comparaison entre les économies française et anglaise ${ }^{78}$. Ce sont ces premières strates du discours sur le progrès, sur l'amélioration, sur les villes des Lumières, sur la révolution industrielle, qui mobilisent la recherche aujourd'hui, tant ces discours ont nourri l'imaginaire des historiens eux-mêmes pendant des générations.

Les rhétoriques des contemporains sont en demiteinte et dans ces regards croisés, les jeux de miroir sont

76 A. Grelon, «Du bon usage du modèle étranger », op. cit., p. 21. Voir également : Robert Fox, « Un enseignement pour une nouvelle ère : le Conservatoire des arts et métiers, 1815-1830 », Cahiers d'histoire du Cnam, 1, 1992, pp. 75-92.

77 M. Aymard, « Histoire et comparaison », op. cit., p. 277. 78 Patrick O’Brien, Giorgio Riello, « Reconstructing the Industrial Revolution : analyses, perceptions and conceptions of Britain's precocious transition to Europe's first industrial society », Londres, London School of Economics, Working papers in economic history, 2004. fréquents. Pour les économistes français du début du XIX'e siècle, le détour par l'Angleterre sert au bilan de la Révolution française, tant « le parallèle avec la Grande-Bretagne, rythmé depuis toujours par des vagues successives d'anglophilie et de phobies patriotiques, constitue [...] un exercice [...] décisif pour juger des effets économiques révolutionnaires $»^{79}$. Régulièrement, au fil des conflits, "la connaissance objective se tarit ${ }^{80}$, les vues des analystes se font sélectives et subjectives, les arguments oscillent entre ambivalence et dénigrement. Dans les enquêtes des analystes, de part et d'autre de la Manche, les ambitions scientifiques sont débordées par les impressions, les peurs et l'investissement symbolique. Dès le milieu du XVIII siècle, les voyageurs dénoncent la condition des artisans anglais soumis aux pressions du négoce, à l'intensification du travail. Au XIXe siècle, la hantise de l'impérialisme anglais, de sa puissance militaire, alimente la légende noire du machinisme (nécessairement anglais) ${ }^{81}$ et de la révolution industrielle dont le concept naît en France, sous la Restauration, du débat sur la question des machines ${ }^{82}$. S'y ajoute la dénonciation par les économistes français (et les ingénieurs, comme le montre K. Chatzis) d'un paupérisme associé à la survivance de l'aristocratie et à la rigidité de la déférence sociale ${ }^{83}$ - des traits de l'identité britannique aussi considérés comme les sources de la stabilité sociale outre-Manche, rappellent G. Riello et P. O'Brien. Seul I'avènement du libre-échange finit par restaurer un discours favorable au succès

79 J.-C. Perrot, « Les effets économiques de la Révolution. Trente années de bilan (1795-1825) », op. cit., p. 453.

$80 \mathrm{lbid}$, p. 455.

81 Sur l'association entre l'Angleterre et les machines, et I'anglophobie qui en résulte, notamment dans une France encore rurale et artisanale au milieu du XIXe siècle, F. Jarrige, "Autour des résistances ouvrières au machinisme », op. cit., pp. 347-350 et id., " Dire le refus des machines : pétitions ouvrières et représentations de l'ordre économique en France, en 1848 », Annales des mines. Série réalités industrielles, février 2009, pp. 49-55, p. 55.

82 F. Jarrige, Au temps des "Tueuses de Bras», op. cit. ; G. S. Jones, "The genesis of the 'Industrial Revolution' », op. cit. ; id., " National bankruptcy and social revolution: European observers on Britain, 1813-1844 », dans Patrick O'Brien et Donald Winch éd., The political economy of British historical experience, 1688-1914, Oxford, Oxford university press, 2002, pp. 61-93.

83 G. S. Jones, "The genesis of the 'Industrial Revolution' », op. cit. ; id., « National bankruptcy and social revolution », op. cit. 
économique anglais en France, alors qu'en Angleterre commence à poindre une critique des conséquences sociales de l'industrialisation, suscitant diverses interprétations, selon les groupes sociaux, des notions de progrès, d'invention et de « révolution industrielle » à la fin du siècle ${ }^{84}$.

Du milieu du XVIII siècle aux années 1860, les enjeux du comparatisme sont guidés par un patriotisme grandissant, comme l'a montré Christine MacLeod ${ }^{85}$. Les techniques, et notamment les inventions, participent de I'imaginaire national des deux côtés de la Manche, de la définition des identités nationales et des stéréotypes, au service d'intérêts et d'usages multiples. " La nationalisation des objets techniques», accompagnée de xénophobie, a constitué en France, selon F. Jarrige, " un langage de substitution » quand I'opposition aux machines est devenue plus difficile à exprimer ${ }^{86}$. Du côté anglais, M. Thébaud-Sorger explique qu'en matière d'aérostation, "défranciser » une invention est déjà un enjeu dans l'Angleterre hanovrienne, avant même que ne se déploie l'exaltation des inventeurs à l'ère victorienne, analysée par C. MacLeod. Les premiers vols en ballon, en 1783, sont investis de messages politiques forts, ils sont soutenus par la monarchie, l'Académie des sciences et les élites éclairées, fédérées dans le culte du progrès ; ils suscitent rapidement l'intérêt de publics élargis pour des attractions spectaculaires qui sont aussi des « fêtes patriotiques locales ». Ce sont aussi de véritables armes diplomatiques comme le suggèrent les premières expériences à Versailles, au moment de la signature du Traité de Paris (1783). L'auteure note que la Révolution fera ensuite du ballon à hydrogène " un outil à la fois technique (observation militaire) et idéologique aux yeux des puissances adverses ». Dès 1784, le vol de Vincenzo Lunardi à Londres donne aux Anglais I'occasion de célébrer à leur tour l'héroïsme d'un aéronaute ${ }^{87}$. La concurrence recouvre à la fois une course au progrès et une guerre des symboles et des investissements politiques. Si le dénigrement sarcastique de l'invention futile, à l'image de l'oisiveté aristocratique, est bien connu, M. Thébaud-Sorger met en lumière le désir des Anglais de se l'approprier et leur pugnacité. Elle rappelle que pour les anglophiles comme Georg Christoph Lichtenberg, professeur de physique à Göttingen, I'aérostation est un bienfait pour l'humanité et non seulement pour la France. Surtout, réfutant une historiographie qui a insisté sur le dédain et la passivité de la Royal Society

84 C. Macleod, Heroes of invention, op. cit., pp. 6-14, 143144 ; J. Vincent, « Cycle ou catastrophe ?», op. cit.

85 C. Macleod, Heroes of invention, op. cit., chap. 3.

86 F. Jarrige, Au temps des « Tueuses de Bras », op. cit., pp. 354-356.

87 C. Macleod, Heroes of invention, op. cit., p. 61. dans ce domaine, l'auteure met en lumière l'existence "d'un milieu savant réactif outre-Manche, qui mêle des hommes de science originaires du continent et de Grande-Bretagne, en lien avec des entrepreneurs, et qui ne néglige pas la découverte ». Les Anglais sont prompts à « se rapproprier la technique aérienne de manière offensive », symboliquement (I'Union Jack du ballon de Lunardi) et intellectuellement (le traité de Tiberius Cavallo revendiquant une généalogie britannique).

À quelques années de distance, ce volontarisme fait écho aux injonctions de Babbage pour encourager l'intérêt britannique pour l'analyse mathématique, comme le rappelle M.-J. Durand-Richard: " découvert par Fermat, rendu analytique par Newton et enrichi par Leibniz, il semblait que le nouveau calcul dût aspirer aux fins les plus hautes. Mais, comme si le sol de ce pays n'était pas propice à sa culture, il s'évanouit bientôt [...], et nous devons maintenant réimporter cet exotisme ». Symétriquement, en France, explique K. Chatzis, les ingénieurs chargés du service des eaux à Paris n'ont de cesse d'ériger leurs choix techniques en école et en modèle, une fois passée l'admiration du système anglais. La gestion urbaine de l'eau fait " désormais partie de l'identité culturelle de la ville, en participant, telle une arme symbolique, à la compétition à laquelle se livrent les capitales européennes en termes de rayonnement culturel». De manière symptomatique, l'ingénieur Édouard Couche note : il « est même assez curieux que, dans l'étude d'un service exclusivement technique, on trouve ainsi, à chaque pas, le reflet du caractère des deux pays ».

L'étude serait à mener sur le long terme, depuis la Renaissance sûrement, pour comprendre les liens qui unissent le génie des peuples et l'ingenium. Au

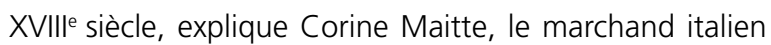
francophile Moccafy, associe les nouveautés tinctoriales françaises (qui manquent aux Anglais) à l'organisation du travail, mais aussi au " génie de la nation » car «si un patron français propose quelque essai à son ouvrier, celui-ci emploiera sa main-d'œuvre pour rien, à cause du génie inné de la nation pour les nouveautés en toutes choses » ${ }^{88}$. Dans les mémoires des officiers de Marine français étudiés par S. Llinares, comme celui du comte de Kersaint, s'exprime la fierté du «génie des inventions » qui fait la «supériorité » de la « nation» française dans la conception des navires. Mais I'apologie est ambivalente, elle sert aussi à souligner les atouts de I'adversaire : I'habileté dans la construction, la finition, la solidité, toutes qualités que possèdent les Anglais et leurs

88 C. Maitte, «Au cœur des manufactures lainières européennes du XVIII" siècle », op. cit. 


\section{Technique, économie et politique entre la France et l'Angleterre (XVIIe-XIXe siècles)}

mécaniciens. Dans l'opposition du «bien conçu » au « bien construit », se lit celle de deux cultures inventives, l'une héritière du projet architectural, de la performance, l'autre marquant le succès des économies de moyens, de la rationalisation des assemblages et de la quête des perfectionnements - en somme, le progrès. La question du génie est indissociable de la « crise de l'évaluation des avantages » au XVIII siècle et pose la question des intérêts, particuliers, généraux. À qui sert le progrès? "la société des hommes ou la nation » ${ }^{89}$ ? Hélène Vérin a montré la réponse sans détour de l'officier de Marine Du Maitz de Goimpy qui relègue « le projet universaliste » (celui de Roland de la Platière, des encyclopédistes) au profit de «la concurrence des nations».

Au XIXe siècle, les idéologies techniciennes accompagnent l'apologie du génie national, de la grandeur des peuples, de la mission civilisatrice des empires à mesure que l'information économique et technique gagne des publics élargis. Au cours de ce procès de publicisation, les arguments deviennent plus foisonnants et complexes. Dans le registre des politiques économiques, Dominique Margairaz a souligné les contradictions qui accompagnent la lutte contre l'Angleterre, dans la politique manufacturière de François de Neufchâteau ${ }^{90}$. À un autre niveau, celui des pratiques commerciales, au cœur même de la Révolution et du Paris sans-culottes, l'éloge des techniques anglaises ne faiblit pas, comme I'illustre le prospectus de l'entrepreneur parisien Le PetitWalle, gratifié et installé par l'État dans des locaux aux Quinze-Vingts. Orné des insignes de la République (pour une «Manufacture Nationale »), le tract vante ses rasoirs d'acier fin à l'imitation de l'Angleterre, dans la langue de l'ennemi (« The only Manufacture in France of good Razors of melted steel in the same manner as that of Sheffield in Yorkshire $\left.{ }^{9}\right)^{91}$. On peut aussi verser au dossier les ambiguïtés qui traversent les institutions savantes, telles les sociétés d'encouragement et l'Institution of civil engineers (adepte de l'ingénierie française, comme le montre B. Marsden), tendues entre des politiques d'échanges héritées de la République des lettres et les rivalités nationales et coloniales. La Society of Arts de Londres, au cœur d'actifs réseaux de diffusion des savoirs techniques au XVIII' siècle, notamment dans les milieux agronomiques, déploie une politique de concurrence des produits français via l'imitation et les substituts $d^{\prime}$ importation ${ }^{92}$. Cette ambivalence s'exprime aussi dans

89 H. Vérin, La gloire des ingénieurs, op. cit., p. 381.

90 Dominique Margairaz, François de Neufchâteau, Biographie intellectuelle, Paris, Publications de la Sorbonne, 2005, pp. 340-341. 91 Archives nationales (Paris), F/12/2224.

92 Liliane Hilaire-Pérez, L'invention technique au siècle des Lumières, Paris, Albin Michel, 2000, chap. 5 ; M. Berg, Luxury les expositions des produits de l'industrie placées sous le signe du mérite de l'inventeur et du patriotisme de I'entrepreneur ${ }^{93}$, puis dans les expositions universelles où s'affirment aussi bien les tensions nationalistes et impérialistes que la figure de l'inventeur bienfaiteur de I'humanité, sous l'égide du libre-échange et de la concorde entre les peuples - une dualité qui sous-tend la patrimonialisation des techniques en Angleterre, dans le sillage du Crystal Palace (Patent Office, Patent Museum) ${ }^{94}$. Dans sa contribution, P. Brenni souligne la perception aiguë par les Anglais, de la concurrence française dans le domaine de l'instrumentation scientifique - jusque dans la constitution des collections scientifiques, y compris celles de Grande-Bretagne - et note le recours au vocabulaire de la course : «I'Exposition a montré [...] que le rythme de progrès industriel de bien des nations européennes, même celles à notre traîne, était plus rapide que le nôtre, et il n'est pas besoin d'être grand clerc pour percevoir que dans une longue course, le navire le plus rapide vaincra », prévient Lyon Playfair. Les expositions, on le sait, jouent un rôle clef dans le regard critique de chaque industrie nationale sur elle-même.

Les jeux de miroir, comme le souligne K. Chatzis, expriment « des visions d'autrui, et par là, des visions de soi-même ». Dans les milieux progressistes britanniques, la science des ingénieurs française est finalement érigée en modèle pour appuyer la promotion de l'industrie dans I'enseignement scientifique outre-Manche. Deux articles illustrent clairement cette tendance. M.-J. DurandRichard explique que l'enjeu pour Babbage, qui regarde vers la France, est de souligner le dynamisme des savants industrialistes français (acquis à la science analytique des ingénieurs modernes) et de critiquer le conservatisme de la science académique anglaise. Non sans écho, B. Marsden montre tout l'intérêt de milieux novateurs tels que I'Institution of civil engineers et les professeurs de génie civil britanniques pour les sciences appliquées françaises dans les années 1830. Une décennie plus tard, le Mechanic's Magazine, journal radical, presse ses lecteurs de s'informer sur la littérature technique française et publie les rapports d'ingénieurs français, parfois cependant égratignés pour leur dogmatisme. Loin de trancher entre rhétoriques pro et anti-françaises, dans ce journal destiné aux artisans, ouvert aux débats et aux polémiques, "la 'France' est une tapisserie à la texture complexe, tissée des fils de la politique

and pleasure, op. cit., pp. 95-103.

93 D. Margairaz, François de Neufchâteau, op. cit., p. 340.

94 C. MacLeod, Heroes of invention, op. cit., chap. 8 et 9 ; John Liffen, «Le Patent Office Museum, 1857-1883, précurseur du Science Museum de Londres ", La Revue du musée des arts et métiers, 51/52, 2010, pp. 56-67. 
mécanicienne ». Les stéréotypes nationaux associés à la mémoire des inventeurs servent des enjeux politiques et identitaires - ceux des travailleurs qualifiés, en quête de reconnaissance. Pour le Mechanic's Magazine, actif soutien du culte naissant de James Watt (considéré comme un exemple du self-help, face à la corruption des élites politiques ${ }^{95}$, la France revêt ainsi un double visage. Elle est le pays, catholique et despotique, où fut persécuté I'artisan protestant Bernard de Palissy, où les expositions des produits de l'industrie célèbrent le luxe aristocratique et négligent les «besoins réels » du pays - un détour, explique l'auteur, pour fustiger la corruption des classes dominantes anglaises. Mais - autre détour - la France est aussi la nation républicaine dans laquelle l'ingéniosité technique est soutenue par des savants acquis au progrès industriel, tel Charles Dupin, encensé dans le Mechanic's Magazine comme éducateur des classes populaires (sur le modèle des Mechanics' Institutes) et comme apologue de l'économie britannique. Le journal se fait le passeur des savoirs technologiques français, y compris dans leur langue d'origine. Pour B. Marsden, «les artisans britanniques, qui lisent le Mechanic's Magazine et lui écrivent, cherchent à consolider à la fois leur identité et leur savoir spécialisé ». Les usages contradictoires de la « France » que livre ce journal polémique les aident à s'affirmer en ce sens. On pourrait compléter le tableau en étudiant les usages en France, de la mythologie technicienne britannique, comme l'a fait F. Jarrige, repérant le culte de James Watt dans le Voyage en Icarie du communiste Etienne Cabet (1840) ${ }^{96}$.

\section{Économie, technologie et comparatisme}

Au fil de ces balancements et de ces contradictions, se construit, à de multiples niveaux, un espace de réflexion fondé sur l'exercice comparatif. Si dans les récits des contemporains, les visées explicatives sont minoritaires par rapport aux argumentaires politiques et sociaux, si la subjectivité l'emporte sur l'objectivité comme l'a montré Jean-Claude Perrot, le parallèle entre la France et l'Angleterre nourrit la science économique. " C'est le nationalisme même qui invite à comprendre les causes de la supériorité de l'adversaire ${ }^{97}$, rappelle François Vatin. G. Riello et P. O’Brien signalent, dans ce volume, les initiatives $d$ 'intellectuels pour bannir les impressions et les peurs face à l'industrialisation britannique et favoriser l'analyse des causes de la croissance. La comparaison favorise la collecte des données et suscite

95 C. MacLeod, Heroes of invention, op. cit. pp. 88-90, 153-154. 96 François Jarrige, « 'Des machines à l'infini'. Le communisme icarien et l'imaginaire utopique des techniques (1830-1848) ", Hypothèses, 2006, pp. 189-208, p. 202.

97 F. Vatin, "Modèle et contre-modèle anglais », op. cit., p. 70. la construction d'outils d'analyse, telle la recherche de fonctions économiques par Blanqui, lui-même enquêteur et lecteur de la presse anglaise, préconisant de construire " une 'physiologie' du corps économique et social $»^{98}$. Le climat de tension avec l'Angleterre n'est pas étranger aux motivations de l'effort statistique dès le Directoire, en vue "d'alimenter la réflexion et les spéculations des entrepreneurs », à l'heure de la prohibition des marchandises anglaises en Espagne, comme l'a rappelé Dominique Margairaz ${ }^{99}$. Peu après, c'est encore le parallèle avec l'Angleterre qui suscite la statistique comparée des «forces productives » et «du produit de ces forces» par Dupin, en vue de révéler " des rapports ignorés» et de " connaître les causes de ces rapports variés et de ces différences »; ainsi se constituent des « évaluations économiques fiables de la force économique comparée des pays ${ }^{100}$. Les expositions universelles ne sont pas sans impact sur l'analyse comparative des voies de I'industrialisation ; pour P. Brenni, le Crystal Palace permet «pour la première fois une comparaison directe et immédiate » entre fabricants français et britanniques et remet en cause la réputation de supériorité des seconds, suscitant une prise de conscience des carences outreManche (et des tentatives d'explication). Selon Blanqui, I'Exposition signait la "supériorité française » en termes de qualité ; « l'observation comparée des deux économies [...] montrait la complémentarité des deux nations », écrit Francis Démier ${ }^{101}$.

Chez les analystes, la comparaison ouvre peu à peu la voie à la compréhension des économies complexes, intégrées (où s'affirment le facteur technique et les notions de «travail » et de «force $»)^{102}$, conduisant à interroger les outils de mesure, les critères d'objectivité. À travers ce comparatisme, se dessinent progressivement « deux conceptions des sciences de la société » ${ }^{103}$ : I'une marquée par l' « interdépendance des facteurs » chez les Français, I'autre affirmant I' « autonomie de I'analyse

98 F. Démier, « Adolphe Blanqui, un 'Libéral critique' », op. cit., p. 67. 99 Dominique Margairaz, «Introduction. De Colbert à la statistique générale de la France », dans D. Margairaz, P. Minard éd., L'information économique XVk-XIXe siècle, op. cit., pp. 143-153, p. 143.

100 Philippe Steiner, "Charles Dupin et Jean-Baptiste Say : arithmétique politique et économie politique », dans C. Christen et F. Vatin éd., Charles Dupin (1784-1873), op. cit., p. 149-163. $101 \mathrm{~F}$. Démier, «Adolphe Blanqui : la leçon anglaise », op. cit., p. 65 ; sur ce thème, Whitney Walton, France at the Crystal Palace. Bourgeois taste and artisan manufacture in the nineteenth century, Berkeley, California university press, 1992.

102 G. S. Jones, "The genesis of the 'Industrial Revolution' », op. cit., p. 227-230; P. Steiner, « Charles Dupin et Jean-Baptiste Say », op. cit., pp. 150-151.

$103 \mathrm{~J}$.-C. Perrot, "Les effets économiques de la Révolution. Trente années de bilan (1795-1825) », op. cit., p. 458. 


\section{Technique, économie et politique entre la France et l'Angleterre (XVIle-XIXe siècles)}

économique » chez les Anglais. Sur le terrain, l'approche française se marque par «la crise de l'évaluation des avantages», soit "l'introduction de considérations de philosophie politique et d'économie politique $»^{104}$. Dans les mémoires d'enquêtes des officiers de la Marine analysés par S. Llinares, au « bilan mesuré » et empirique des avantages et des inconvénients de telle technique, succède l'étude de l'organisation des chantiers, de I'administration de la Navy, de la formation de la main d'œuvre et des capacités inventives mobilisant tous les secteurs de l'économie (cuivre, cordages, pompes). Sur le plan théorique, la tension entre les deux approches est perceptible à travers I'hostilité à l'égard de la théorisation ricardienne dans l'enseignement de Blanqui au Conservatoire des arts et métiers ${ }^{105}$; elle se poursuit dans le rejet de l'école économique anglaise par Frédéric Le Play, au profit d'une "compréhension intégrée des initiatives humaines », véritable science sociale de I'action et des rationalités pratiques, fondée sur une « anthropologie économique comparative ${ }^{106}$.

On perçoit ici l'un des rameaux de la technologie, comme science de l'homme. Qu'en est-il en effet de la science technologique entre la France et l'Angleterre ? Quel est le rôle de ce comparatisme dans l'émergence d'une science de la technique, née sur le continent, au tournant des Lumières et de la révolution industrielle, dans le sillage de l'économie politique ${ }^{107}$ ? Les termes euxmêmes, technologie et technology, qui commencent à se dissocier, méritent de s'interroger sur l'histoire comparée de la discipline. On se rappelle qu'en 1855, à l'université d'Edinbourg, George Wilson, directeur du Musée industriel écossais, qui intitule sa conférence inaugurale à l'université d'Edinbourg «What is technology ? »108,

104 H. Vérin, La gloire des ingénieurs, op. cit., p. 379.

105 F. Démier, « Adolphe Blanqui, un 'Libéral critique' », op. cit., p. 63. 106 Alain Cottereau, «Statistique et critique de l'économie politique. Le Play, économiste ", dans Stéphane Baciocchi, Jérôme David éd., Frédéric Le Play. Anthologie et correspondance, Les études sociales, 142-143-144, II-2005/2006, pp. 119131. Sur la méthode comparative de Le Play, voir aussi JeanFrançois Belhoste, «Pourquoi Frédéric Le Play, métallurgiste, explorateur de la Russie, s'intéressa à la question sociale », dans J.-F. Belhoste, S. Benoît, S. Chassagne, P. Mioche éd., Autour de I'industrie, op. cit., pp. 3-21.

107 Hélène Vérin, « La technologie : science autonome ou science intermédiaire ", Documents pour l'histoire des techniques, 14, 2007, pp. 134-143.

108 Robert G. W. Anderson, «' What is technology?': edu-cation through museums in the mid-nineteenth century », British journal for the history of science, 1992, xxxv, pp. 169-184; George Wilson, What is technology? An inaugural lecture delivered in the University of Edinburgh on November 7, 1855, Edinburgh, Sutherland \& Knox, et Londres, Simpkin, Marshall \& Co., 1855. note que ce terme est «si peu familier aux oreilles anglaises "109 qu'il lui faut expliquer le programme qu'il propose en marge de la science universitaire: l'étude des arts industriels, classés par opérations et matériaux, illustrés par les collections de modèles et les visites d'entreprises (qui attirent un public ouvrier) - une définition imprégnée du modèle technologique et civique français et qui ne se confond pas avec celle $d^{\prime}$ " applied science », "circonlocution maladroite pour art ou pratique ${ }^{110}$. La technologie a bien été une discipline enseignée outre-Manche, du moins en Ecosse, comme I'illustre aussi William Rankine, professeur de génie civil et mécanique à Glasgow au milieu du siècle, tentant de défendre la science naissante de la technologie face à la science académique ${ }^{111}$. L'histoire de la technologie, dans une dimension comparative, en est à ses débuts et le présent ouvrage en offre les prémices.

Plusieurs chapitres permettent d'ouvrir des perspectives nouvelles, qu'il s'agisse de la comparaison des contenus de la technologie, termes à termes, ou de l'analyse des ressorts du comparatisme technique. L'analyse comparée pionnière, par Joost Mertens, des dictionnaires d'Andrew Ure (1839-1853) et de Louis-Sébastien Lenormand (18221835) révèle de fortes différences. L'auteur décèle chez I'Anglais une vision technologique radicale, sélective et utopique, réduite au processus manufacturier, fondée sur la description des procédés de transformation des matières premières, par voie mécanique ou chimique, en des « objets généraux de valeur échangeable » (« general objects of exchangeable value »112. À I'inverse, le Français

109 «...this word in this sense, is not familiar to an English ear ». On notera la symétrie avec le mot «entrepreneur »: Hélène Vérin rappelle que John Stuart Mill regrettait que ce mot ne soit pas familier en Angleterre; Entrepreneurs, entreprise. Histoire d'une idée, Paris, PUF, 1982, p. 10.

110 C. Macleod, Heroes of invention, op. cit., p. 360 ( « a clumsy circumlocution for art or practice $»)$.

111 Ben Marsden, "Engineering science in Glasgow: economy, efficiency and measurement as prime movers in the differenciation of an academic discipline », British journal for the history of science, 25, 1992, pp. 319-346

112 Louis-Sébastien Lenormand et Louis-Benjamin Francœur, Dictionnaire technologique, ou nouveau dictionnaire universel des arts et métiers, et de l'économie industrielle et commerciale, Paris, Thomine \& Fortic, 1822-1835 ; Andrew Ure, Dictionary of arts, manufactures, and mines, containing a clear exposition of their principles and practice (1839), Londres, Longman, 1853.(4e éd.). Voir aussi Joost Mertens, "The Annales de l'industrie (1820-1827): a technological laboratory for the industrial modernization of France », History and technology, vol. 20, n², juin 2004, pp. 135-163; id., " Technology as a science of the industrial arts: Louis-Sébastien Lenormand (1757-1837) and the popularization of technology », History and technology, vol. 18, n³, 2002, pp. 203-231 ; Jan Sebestik, « Les commencements de la technologie Postface/Préface ? ", Documents pour l'histoire des 
propose une logique inclusive, systémique, intégrant et connectant les sphères des activités économiques, dans le droit fil du sens originel (et hautement politique) du mot « industrie », tel que l'emploient Jean-Baptiste Say dans son Traité d'économie politique de $1803^{113}$, Dupin dans Le petit producteur français (1827) ${ }^{114}$ ou Chaptal dans De l'industrie française (1819) et comme fondateur de la Société d'encouragement pour l'industrie nationale. Chez Blanqui (comme chez Dupin), le propos se double d'une apologie radicale de la petite entreprise et des « innombrables métiers qui permettent à l'ouvrier ... de déployer toutes les facultés de son intelligence, de vivre de la vie de famille et non pas comme un automate, d'être un homme et non une machine .... ${ }^{115}$. La technologie générale, dans la lignée de l'Entwurf der allgemeinen Technologie de Johannes Beckmann (1806), est définie en France comme l'étude des arts et manufactures, décrits en une langue unifiée et classés («réduits ») de manière "systématique », en opérations, afin de promouvoir, par contiguïté ou par transversalité entre métiers, les combinaisons inventives - un procès culturel et politique de publicisation des savoirs artisanaux, qui s'affirme à travers l'ambition civique et pédagogique de cette science sous la Révolution ${ }^{116}$. Les liens sont évidents avec une économie politique née dans le creuset des savoirs administratifs, au service du bien public, comme le montrent $C$. Demeulenaere-Douyère et $D$. J. Sturdy dans leur analyse de l'Enquête du Régent ${ }^{117}$. Si ce canal n'est pas le seul, l'information technologique prend forme et revêt une rationalité qui tient déjà à l'ambition d'exhaustivité, au cœur même des dispositifs gouvernementaux. Le recensement des ressources constitue l'une des matrices

techniques, 14, 2007, p. 123-133.

113 G. S. Jones, "The genesis of the 'Industrial Revolution' », op. cit., p. 214 ; id., "Saint-Simon and the liberal origins», op. cit., pp. 29-37.

114 François Vatin, « Les aventures du bon Monsieur Lerond, le petit fabricant français », dans C. Christen et F. Vatin éd., Charles Dupin (1784-1873), op. cit., p. 207-221.

115 F. Démier, "Adolphe Blanqui, un 'Libéral critique' », op. cit., p. 71 ; id., « Adolphe Blanqui : la leçon anglaise », op. cit., pp. 59-60. $116 \mathrm{~J}$. Mertens, «Technology as a science of the industrial arts », op. cit. ; id., " Éclairer les arts : Eugène Julia de Fontenelle (1780-1842), ses manuels Roret et la pénétration des sciences appliquées dans les arts et manufactures ", Documents pour I'histoire des techniques, 18, 2009, p. 95-112.

117 Outre leur article dans le présent volume: Christiane Demeulenaere-Douyère et David J. Sturdyéd., L'Enquête du Régent 1716-1718. Sciences, techniques et politique dans la France préindustrielle, Turnhout, Brepols, 2008 ; C. Demeulenaere-Douyère, "Une tentative d'inventaire des richesses minières de la France : I'Enquête du Régent, 1716-1718 ", Documents pour l'histoire des techniques, 16, 2008, pp. 9-16. de la science technologique, via l'entreprise de stabilisation et de réduction des langues et la mise en place d'une vision globale, connectée, sectorielle des activités, au nom du bien public (notamment par l'insistance sur les richesses minières, les matières premières). L'approche inclusive, qui fait une large place aux gestes de métier, érigés en « génie » français lors de l'exposition de 1851 les « industries naturelles ${ }^{118}$, soit " les arts sur lesquels I'habileté de la main et la pureté du goût peuvent exercer leur influence ", selon Blanquil ${ }^{19}$ - se double d'une vision technologique, portée par le saint-simonisme comme l'a montré Antoine Picon : " en rendant explicites les liens qui unissent les différentes branches de la production, la technologie doit permettre de maîtriser les hybrides qui se font jour aux frontières des domaines techniques traditionnels » (comme les chemins de fer) ${ }^{120}$. En France, se dessinent ainsi des rameaux de la « technologie, science humaine ».

En Angleterre, si la technologie comme science des arts, et non seulement des processus industriels, s'exprime aussi (même chez un technologue "radical » comme Charles Babbage, dont la pensée reste marquée par une économie du produit qui a fait l'originalité de

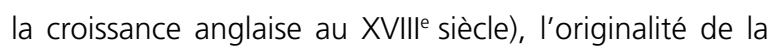
philosophy of manufactures est de fournir à la fois une " économie morale du système automatique » comme le dit Ure, soit la justification du machinisme (F. Jarrige), et une théorisation de la technique, une technologie, qui se donne pour but d'abstraire, de synthétiser le travail en opérations, transférées des humains aux machines, en vue du meilleur rendement. De ce côté de la Manche, la technologie "commence au moment où I'homme et la machine échangent leurs fonctions ${ }^{121}$. Cette signification nouvelle, "restrictive » comme la désigne J. Mertens, d'une science de I' « operative industry » (A. Ure), s'affirme pleinement en Grande-Bretagne dès les années 1830. Elle se déploie en France dans la deuxième moitié du siècle et signe la fin de la technologie générale ${ }^{122}$. La «question des machines », au cœur de l'économie des manufactures, ouvre sur l'abstraction du

118 Voir le rôle du mélange du naturel et de l'artificiel dans la définition de la modernité au Crystal Palace : Antoine Picon, Les saintsimoniens. Raison, imaginaire, utopie, Paris, Belin, 2002, p. 218.

119 F. Démier, « Adolphe Blanqui : la leçon anglaise », op. cit., p. 66. 120 A. Picon, Les saint-simoniens, op. cit., pp. 206-207.

121 Jacques Guillerme, Jan Sebestik, «Les commencements de la technologie » Thalès, 1968, xii, pp. 1-72, réédité dans Documents pour l'histoire des techniques, 14, 2007, pp. 49-122, p. 111.

122 Joost Mertens, «Le déclin de la technologie générale : Léon Lalanne et l'ascendance de la science des machines », Documents pour l'histoire des techniques, 20, 2010, sous presse. Nous remercions vivement l'auteur pour la communication de ce texte. 


\section{Technique, économie et politique entre la France et l'Angleterre (XVIIe-XIXe siècles)}

travail comme unité de mesure de la valeur d'échange et justifie la critique marxienne de la «technologie». La «technologie » est pour Marx, cette «science toute moderne » qui se donne en effet pour principe « de dissocier tout procès de production », « sans aucun égard pour la main humaine en ses éléments constitutifs » et de mettre à nu « les quelques formes fondamentales du mouvement selon lesquelles, malgré toute la variété des instruments utilisés, toute action productive du corps humain procède nécessairement $»^{123}$.

On perçoit bien sûr déjà l'expression de cette technologie industrielle (et "restrictive ») en France, dans le Plan de technonomie de Gérard-Joseph Christian en 1819 qui distingue le «travail industriel proprement dit », comme le souligne justement J. Mertens. C'est aussi la science analytique des ingénieurs français qui promeut le calcul du travail, de l'effet utile ou « quantité d'action », " des productivités relatives de chaînes opératoires complexes $»^{124}$. Symétriquement, rappelons

123 Karl Marx, Le capital. Critique de l'économie politique, Livre premier. Le procès de production du capital (1867), $4^{e}$ édition allemande (1890), Paris, Presses universitaires de France, Quadrige, 1993, p. 546. Marx commence la rédaction de la Contribution à la critique de l'économie politique en 1857. La traduction anglaise du premier tome paraît en 1887. Sur la réception de la pensée marxiste en Angleterre, voir l'analyse de Christine MacLeod, Heroes of invention, op. cit., pp. 276-277. L'auteure rappelle que l'invention principale pour Marx n'est pas la machine à vapeur mais la machine-outil qui supprime l'habileté du geste de l'ouvrier et donc prive celui-ci de son pouvoir économique. Pour Marx, «La machine à vapeur proprement dite [...] n'a provoqué aucune révolution industrielle. C'est à l'inverse beaucoup plus la création des machines-outils qui a rendu nécessaire la révolution de la machine à vapeur [...] La machine, qui est à la base de la révolution industrielle, remplace l'ouvrier manipulant son outil singulier, pas un mécanisme qui opère en une fois avec quantité de ces outils ou d'outils de même espèce... » (p. 421).

124 Jacques Guillerme, "A propos du concept de rendement », Actes du XIl' congrès international d'histoire des sciences, Paris, Blanchard, 1971, p. 83-87, rééd. dans Jacques Guillerme, L'art du projet. Histoire, technique, architecture, textes réunis par Hélène Vérin et Valérie Nègre, Wavre, Mardaga, 2008, pp. 6972; A. Picon, L'invention de l'ingénieur moderne, op. cit. ; Bernard Grall et François Vatin, "La machine et l'impôt : Jules Dupuit, l'économie politique et la mécanique industrielle », dans Jean-Pascal Simonin, François Vatin éd., L'œuvre multiple de Jules Dupuit (1804-1866). Calcul d'ingénieur, analyse économique et pensée sociale, Angers, Presses universitaires d'Angers, 2002, pp. 39-60; Jean-Yves Dupont, "Evaluer la puissance des machines au XIXe siècle », Musée des arts et métiers, La revue, 48,2007 , pp. 42-54. On doit prendre en compte l'histoire du cheval-vapeur et de la détermination d'une valeur abstraite, quantifiable du travail à partir de l'usage démultiplié de la force du cheval : Daniel Roche, La culture équestre de l'Occident XVle- les ambiguïtés de Babbage et l'influence de la technologie française sur sa pensée. D'une part, «la conception que se fait Charles Babbage des sciences mécaniques est inséparable d'une réflexion philosophique sur l'histoire et sur la société, d'une conception de l'homme de science dans laquelle mathématiques, théologie, sciences naturelles, mais aussi sciences morales et politiques ne sont pas séparées ${ }^{125}$. D'autre part, M.-J. DurandRichard montre dans ce volume, que pour le groupe des mathématiciens réformateurs de Cambridge, mené par Babbage, la France est un modèle, non seulement pour rationaliser l'activité scientifique, mais aussi pour introduire I'analyse algébrique dans la formation des élites, afin de promouvoir la connaissance des savoirs opératoires, source de gestion et de contrôle de la production et de la machine sociale dans son ensemble. Babbage est l'un des passeurs outre-Manche de cet «idéal analytique » qui a ouvert la voie chez les ingénieurs français à «la modélisation des phénomènes dynamiques » ${ }^{126}$. L'auteure rappelle que, en 1819, Babbage rencontre Pierre-Simon Laplace et Gaspard Riche de Prony dont les travaux nourrissent ses tentatives d'automatisation du calcul, conçu comme "opération de l'esprit ». Babbage en vient à définir l' « opération » comme « tout processus qui transforme la relation mutuelle entre deux choses ou plus », incluant " tous les sujets de l'univers » - une vision technologique qui, par son universalisme, porte une empreinte française. "Babbage envisage ainsi une rationalisation systématique de la science comme de I'industrie à partir de ces méthodes, dans un effort de coordination qui ne peut venir que d'un pouvoir central ». Pour M.-J. Durand-Richard, c'est «l'identification du monde anglo-saxon comme terre du libéralisme » qui a fait écran à la compréhension de la pensée de Babbage, admirateur des écoles d'ingénieurs en France, comme lieux de formation des élites et fleurons de la science analytique, sous l'égide de l'État. Non sans paradoxe, avec Babbage, se profile, via l'apologie du modèle technocratique français ${ }^{127}$, la réhabilitation du savant, moins populaire que l'inventeur dans l'Angleterre victorienne mais à terme vainqueur dans la concurrence des mémoires ${ }^{128}$. Un motif analogue se dessine au même moment avec Betancourt, représentant par excellence

XIXe siècle. L'ombre du cheval, tome 1 : Le cheval moteur, Paris, Fayard, 2008, p. 28-30 (le terme horsepower apparait en 1809). 125 V. Bourdeau, F. Jarrige, J. Vincent, Les luddites, op. cit., p. 69. 126 A. Picon, L'invention de l'ingénieur moderne, op. cit.

127 Bruno Belhoste, La formation d'une technocratie. L'École polytechnique et ses élèves de la Révolution au Second Empire, Paris, Belin, 2003.

128 C. MacLeod, Heroes of invention, op. cit., chap. 12 (« Science and the disappearing inventor »). 
l'expert technique "féru en théories mathématiques» et proposant en Russie, pour l'Institut des ingénieurs des voies de communication, une synthèse de l'École des ponts et chaussées et de l'École polytechnique qui exclut tout autre apport, ni espagnol ni britannique, explique I. Gouzévitch (« ses anciens élèves et collègues espagnols qui I'avaient suivi en Russie ne sont pas sollicités pour enseigner à l'institution russe. Quant aux Britanniques, ils apparaissent d'abord uniquement dans ses transactions entrepreneuriales $»)$.

L'étude de ces chassés-croisés de la science et de la technologie de part et d'autre de la Manche (et dans l'espace de résonance européen) renouvelle les études pionnières des années 1990 consacrées à la comparaison des « 'modèles' distincts de formation des ingénieurs » ${ }^{129}$. C'est la compréhension fine de la culture des passeurs et, plus globalement, des multiples médiations, qui permet peu à peu de comprendre les conditions d'émergence d'une technologie générale dans l'espace franco-anglais, mais aussi la montée en puissance des sciences appliquées et d'une acception étroite de la technologie, comme " science intermédiaire ${ }^{130}$. L'expression est employée par Charles Laboulaye, dans sa troisième traduction du Dictionary of arts, manufactures, and mines de Andrew Ure, en $1873^{131}$. C'est au fil des traductions ${ }^{132}$, explique

129 I. Gouzévitch, A. Grelon et A. Karvar, «Présentation», op. cit., p. 11. Voir également: Antoine Picon, «Technological traditions and national identities. A comparison between France and Great Britain during the XIX ${ }^{\text {th }}$ century », dans Efthymios Nicolaïdis et Konstantinos Chatzis éd., Science, technology and the $X I X^{\text {th }}$ century state, Athènes, Institut de recherches néohelléniques, 2000, pp. 13-21.

$130 \mathrm{H}$. Vérin, "La technologie : science autonome ou science intermédiaire », op. cit.

$131 \mathrm{~J}$. Mertens, "Le déclin de la technologie générale : Léon Lalanne et l'ascendance de la science des machines », op. cit.

132 Sur les enjeux nouveaux de la traduction à la fin du XVIII" siècle, Patrice Bret écrit : "la traduction scientifique peut être une œuvre reconnue, [...] moins par sa capacité à transposer le génie de la langue d'origine dans celle du traducteur, sa fidélité au contenu et son exactitude, que par le simple fait de communiquer des observations ou interprétations nouvelles dans cette dernière langue, [...] et surtout, peut-être, par la valeur ajoutée qu'apportent les additions, corrections et commentaires
J. Mertens, que Laboulaye construit peu à peu sa définition et lui attribue une signification qui s'est affirmée dans les milieux savants français depuis le XVIIIle siècle, en concurrence avec la technologie beckmannienne mais en écho avec le sens nouveau pris par le mot « technology » outre-Manche.

Identités hybrides (des produits, des techniques, des individus et de leurs cultures), aptitudes aux réseaux, essor des moyens d'échanger, montée des craintes face aux transformations économiques, mais aussi déploiement des comparatismes: le colloque croisait trois registres d'analyse, l'étude des causes de l'industrialisation grâce à I'histoire renouvelée des circuits techniques, celle du sens donné par les contemporains aux réalités perçues des deux côtés de la Manche, celle des raisonnements analogiques et de l'objectivité. Si la subjectivité des contemporains, britanniques et étrangers, souvent inquiets de voir leur monde vaciller, éclairait "leur révolution industrielle », elle constituait aussi, parado-xalement, la matrice des méthodes d'analyse fonda-trices des sciences sociales. Histoire des savoirs, des représentations et de l'abstraction : l'espace de réflexion de ce travail collectif inscrivait donc I'histoire des techniques et de l'économie dans celle des pratiques culturelles et de I'histoire intellectuelle. Ainsi, en écho aux propos de G. Riello et P. O'Brien, loin d'opposer I'histoire " des causes et des effets de l'industrialisation, des tendances et des macro-phénomènes » à celle des représentations des contemporains et de leur compréhension située des réalités, nous avons au contraire voulu nous inscrire dans une histoire ouverte, " totale » de l'espace économique trans-Manche, prolongeant le propos de Daniel Roche selon lequel « il est un peu artificiel d'opposer les systèmes de significations et les systèmes de relations, tant les uns et les autres sont liés dans le mouvement qui nous porte à vivre l'interprétation du passé ${ }^{133}$.

de l'auteur, du traducteur et parfois d'autres auteurs »; Mme $P^{* * *}$ de Dijon. Sociabilités savantes, traduction scientifique et spécialisation de la presse au $18^{\mathrm{e}}$ siècle. Nous remercions sincèrement l'auteur de nous avoir permis de lire ce manuscrit inédit. 133 Daniel Roche, "Avant-propos. De I'histoire sociale à I'histoire des cultures : le métier que je fais », dans id. éd., Les Républicains des lettres, Paris, Fayard, 1988, pp. 7-22, p. 22. 